\title{
Improved filters for gravitational waves from inspiraling compact binaries
}

\author{
Thibault Damour \\ Institut des Hautes Etudes Scientifiques, 91440 Bures-sur-Yvette, France \\ and DARC, CNRS-Observatoire de Paris, 92195 Meudon, France \\ Bala R. Iyer \\ Raman Research Institute, Bangalore 560 080, India \\ B. S. Sathyaprakash \\ Cardiff University of Wales, P.O. Box 913, Cardiff, CF2 3YB, United Kingdom \\ and California Institute of Technology, Pasadena, California 91125 \\ (Received 19 August 1997; published 31 December 1997)
}

\begin{abstract}
The order of the post-Newtonian expansion needed to extract in a reliable and accurate manner the fully general relativistic gravitational wave signal from inspiraling compact binaries is explored. A class of approximate wave forms, called $P$-approximants, is constructed based on the following two inputs: (a) the introduction of two new energy-type and flux-type functions $e(v)$ and $f(v)$, respectively, (b) the systematic use of the Padé approximation for constructing successive approximants of $e(v)$ and $f(v)$. The new $P$-approximants are not only more effectual (larger overlaps) and more faithful (smaller biases) than the standard Taylor approximants, but also converge faster and monotonically. The presently available $(v / c)^{5}$-accurate post-Newtonian results can be used to construct $P$-approximate wave forms that provide overlaps with the exact wave form larger than $96.5 \%$, implying that more than $90 \%$ of potential events can be detected with the aid of $P$-approximants as opposed to a mere $10-15 \%$ that would be detectable using standard post-Newtonian approximants.
\end{abstract}

[S0556-2821(98)00104-0]

PACS number(s): 04.30.Db, 04.25.Nx, 04.80.Nn, 95.55.Ym

\section{INTRODUCTION AND METHODOLOGY}

Inspiraling compact binaries consisting of neutron stars and/or black holes are among the most promising candidate sources for interferometric detectors of gravitational waves such as the Laser Interferometric Gravitational Wave Observatory (LIGO) and VIRGO. The inspiral wave form enters the detector bandwidth during the last few minutes of the evolution of the binary. Since the wave form can, in principle, be calculated accurately, it should be possible to track the signal phase and hence enhance the signal-to-noise ratio by integrating the signal for the time during which the signal lasts in the detector band. This is achieved by filtering the detector output with a template which is a copy of the expected signal. Since in general relativity the two-body problem has not been solved, the exact shape of the binary wave form is not known and experimenters intend to use as a template an approximate wave form computed perturbatively with the aid of a post-Newtonian expansion [1-11]. Thus, template wave forms used in detection will be different from the actual signal that may be present in the detector output. As a result the overlap of template and signal wave forms would be less than what one would expect if they had exactly matched.

In this paper we explore the order of the post-Newtonian expansion needed to extract in a reliable and accurate manner the actual, fully general relativistic signal. Previous attacks on this problem $[2,3,11-14]$ suggested that a very high post-Newtonian order (maybe as high as $v^{9} / c^{9}$ beyond the leading approximation) might be needed for a reasonably accurate signal extraction [15]. Our conclusions are much more optimistic. We show that, starting only from the presently known $(v / c)^{5}$-accurate (finite mass) post-Newtonian results [6-10], but using them in a novel way, we can construct new template wave forms having overlaps larger than $96.5 \%$ with the "exact" wave forms. Since a reduction in the signal-to-noise ratio by $3.5 \%$ only results in a loss in the number of events by $10 \%$, and since our computations indicate that the new templates entail only small biases in the estimate of signal parameters (see Tables V and IX below), we conclude that presently known post-Newtonian results will be adequate for many years to come.

Before entering the details of our construction, let us clarify, at the conceptual level, the general methodology of this work. Central to our discussion is the following data analysis problem: On the one hand, we have some exact gravitational wave form $h^{X}\left(t ; \lambda_{k}\right)$ where $\lambda_{k}, k=1, \ldots, n_{\lambda}$, are the parameters of the signal (comprising, notably, the masses $m_{1}$ and $m_{2}$ of the members of the emitting binary [16]). On the other hand, we have theoretical calculations of the motion of [17] and gravitational radiation from [6-10], binary systems of compact bodies (neutron stars or black holes). The latter calculations give the post-Newtonian expansions (expansions in powers of $v / c$ ) of, essentially [18], two physically important functions: an energy function $E(v)$ and a gravitational flux function $F(v)$ (see exact definitions below). Here, the dimensionless argument $v$ is an invariantly defined "velocity" [19] related to the instantaneous gravitational wave frequency $f^{\mathrm{GW}}$ (= twice the orbital frequency) by

$$
v=\left(\pi m f^{\mathrm{GW}}\right)^{1 / 3},
$$




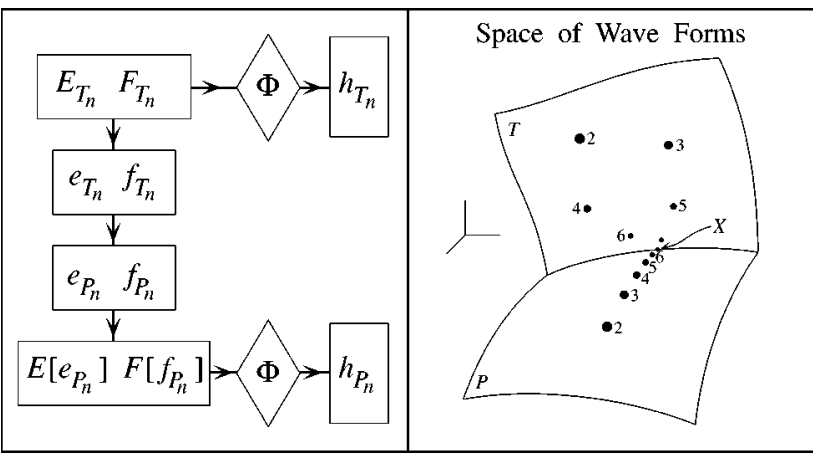

FIG. 1. Schematic illustration of our methodology to compute improved templates.

where $m \equiv m_{1}+m_{2}$ is the total mass of the binary. Let us denote by $E_{T_{n}}$ and $F_{T_{n}}$ the $n^{\text {th }}$-order Taylor approximants of the energy and flux functions:

$$
\begin{aligned}
& E_{T_{n}}=\sum_{k=0}^{n} E_{k}(\eta) v^{k}=E(v)+O\left(v^{n+1}\right), \\
& F_{T_{n}}=\sum_{k=0}^{n} F_{k}(\eta) v^{k}=F(v)+O\left(v^{n+1}\right),
\end{aligned}
$$

where

$$
\eta \equiv \frac{m_{1} m_{2}}{\left(m_{1}+m_{2}\right)^{2}}
$$

is the symmetric mass ratio. For finite $\eta$, the Taylor approximants (1.2), (1.3) are known for $n \leqslant 5$ [17,6-10]. In the test mass limit $\eta \rightarrow 0, E(v)$ is known exactly and $F(v)$ is known up to the order $n=11[1-5,11]$. (There are logarithmic terms appearing for $n \geqslant 6$ that we shall duly discuss later, but in this Introduction we simplify the notation by not introducing them.)

The problem is to construct a sequence of approximate wave forms $h_{n}^{A}\left(t ; \lambda_{k}\right)$, starting from the post-Newtonian expansions (1.2), (1.3). In formal terms, any such construction defines a map from the set of the Taylor coefficients of $E$ and $F$ into the (functional) space of wave forms (see Fig. 1). Up to now, the literature has considered only the most standard map, say $T$,

$$
\left(E_{T_{n}}, F_{T_{n}}\right)_{\rightarrow}^{T} h_{n}^{T}\left(t, \lambda_{k}\right),
$$

obtained by inserting the successive Taylor approximants [20] (1.2), (1.3) into the integral, giving the time evolution of the gravitational wave phase; see e.g. [12,13]. (Details are given below.) In this work, we shall define a new map, say " $P$," based on a four-stage procedure (Fig. 1):

$$
\begin{aligned}
\left(E_{T_{n}}, F_{T_{n}}\right) & \rightarrow\left(e_{T_{n}}, f_{T_{n}}\right) \rightarrow\left(e_{P_{n}}, f_{P_{n}}\right) \rightarrow\left(E\left[e_{P_{n}}\right], F\left[e_{P_{n}}, f_{P_{n}}\right]\right) \\
& \rightarrow h_{n}^{P}\left(t, \lambda_{k}\right) .
\end{aligned}
$$

The two essential ingredients of our procedure are (i) the introduction, on theoretical grounds, of two new, supposedly more basic and hopefully better behaved, energy-type and flux-type functions, say $e(v)$ and $f(v)$, and (ii) the systematic use of Padé approximants (instead of straightforward Taylor expansions) when constructing successive approximants of the intermediate functions $e(v), f(v)$. Let us also note that we further differ from previous attacks on the problem by using a numerical (discrete) fast Fourier transform to compute the overlaps between the exact and approximate wave forms. We find that the previously used analytical stationary phase approximation gives only poor estimates of the overlaps (see Table II).

One of the aims of the present paper is to show that the new sequence of templates $h_{n}^{P}(t ; \lambda)$ is, in several ways, "better' than the standard one $h_{n}^{T}(t ; \lambda)$. In this respect, it is convenient to introduce some terminology. We shall say that a multi-parameter family of approximate wave forms $h^{A}\left(t ; \mu_{k}\right), k=1, \ldots, n_{\mu}$, is an effectual model of some exact wave form $h^{X}\left(t ; \lambda_{k}\right) ; k=1, \ldots, n_{\lambda}$ (where one allows the number of model parameters $n_{\mu}$ to be different from, i.e. in practice, strictly smaller than $\left.n_{\lambda}\right)$, if the overlap, or normalized ambiguity function, between $h^{X}\left(t ; \lambda_{k}\right)$ and the timetranslated family $h^{A}\left(t-\tau ; \mu_{k}\right)$,

$$
\mathcal{A}\left(\lambda_{k}, \mu_{k}\right)=\max _{\tau, \phi} \frac{\left\langle h^{X}(t ; \lambda), h^{A}(t-\tau ; \mu)\right\rangle}{\sqrt{\left\langle h^{X}(t ; \lambda), h^{X}(t ; \lambda)\right\rangle\left\langle h^{A}(t ; \mu), h^{A}(t ; \mu)\right\rangle}},
$$

is, after maximization on the model parameters $\mu_{k}$ [21], larger than some given threshold, e.g. $\max _{\mu_{k}} \mathcal{A}\left(\lambda_{k}, \mu_{k}\right)$ $\geqslant 0.965$ [22]. [In Eq. (1.7) the scalar product $\langle h, g\rangle$ denotes the usual Wiener bilinear form involving the noise spectrum $S_{n}(f)$ (see below).] While an effectual model may be a precious tool for the successful detection of a signal, it may do a poor job in estimating the values of the signal parameters $\lambda_{k}$. We shall then say that a family of approximate wave forms $h^{A}\left(t ; \lambda_{k}^{A}\right)$, where the $\lambda_{k}^{A}$ are now supposed to be in correspondence with (at least a subset of) the signal parameters, is a faithful model of $h^{X}\left(t ; \lambda_{k}\right)$ if the ambiguity function $\mathcal{A}\left(\lambda_{k}, \lambda_{k}^{A}\right)$, Eq. (1.7), is maximized for values of the model parameters $\lambda_{k}^{A}$ which differ from the exact ones $\lambda_{k}$ only by acceptably small biases [23]. A necessary [24] criterion for faithfulness, and one which is very easy to implement in practice, is that the "diagonal" ambiguity $\mathcal{A}\left(\lambda_{k}, \lambda_{k}^{A}=\lambda_{k}\right)$ be larger than, say, 0.965 .

Using this terminology, we shall show in this work that our newly defined map, Eq. (1.6), defines approximants which, for practically all values of $n$ we could test, are both more effectual (larger overlaps) and more faithful (smaller biases) than the standard approximants Eq. (1.5). A related property of the approximants defined by Eq. (1.6) is that the convergence of the sequence $\left(h_{n}^{P}\right)_{n \in \mathcal{N}}$ is both faster and much more monotonous than that of the standard sequence $\left(h_{n}^{T}\right)_{n \in \mathcal{N}}$. This will be shown below in the (formal) test mass limit $\eta \rightarrow 0$ where one knows both the exact functions $E(v)$ and (numerically) $F(v)$ [13], and their Taylor expansions to order $v^{11}$ [11]. The convergence will be studied both "visually" (by plotting successive approximants to $E$ and $F$ ) and "metrically" [by using the ambiguity function (1.7) to define a distance between normalized wave forms]. Most of our convergence tests utilize the rich knowledge of the postNewtonian expansions (1.2), (1.3) in the test mass limit 
$\eta \rightarrow 0$. The very significant qualitative and quantitative advantages of the new sequence of approximants, Eq. (1.6), over the standard one, Eq. (1.5), when $\eta \rightarrow 0$, make it plausible that the new sequence $\left(h_{n}^{P}\right)$ will also fare much better in the finite mass case $0 \neq \eta \leqslant \frac{1}{4}$. This question, which we can call the problem of the robustness of our results under the deformations brought by a finite value of $\eta$ in the coefficients $E_{k}(\eta), F_{k}(\eta)$ in Eqs. (1.2), (1.3), is more difficult to investigate, especially because one does not know, in this case, the "exact" results for $E(v ; \eta)$ and $F(v ; \eta)$. We could, however, check the robustness of our construction in two different ways: (i) by studying the "Cauchy criterion" for the convergence of the (short) sequence $\left[h_{0}^{P}(\eta), h_{2}^{P}(\eta)\right.$, $\left.h_{4}^{P}(\eta), h_{5}^{P}(\eta)\right]$ versus that of the corresponding Taylor sequence, and (ii) by introducing a one-parameter family of fiducial "exact" functions $e_{\kappa_{0}}^{X}(v), f_{\kappa_{0}}^{X}(v)$ to model the unknown higher-order $(n \geqslant 6) \eta$-dependent contributions to the post-Newtonian expansions (1.2), (1.3) and by studying for a range of values of the parameter $\kappa_{0}$ the convergence of the short sequence $\left[h_{0}^{P}(\eta), \ldots, h_{5}^{P}(\eta)\right]$ toward the fiducial "exact' wave form $h_{\kappa_{0}}^{X}(\eta)$.

Though we believe the work presented below establishes the superiority of the new approximants $h_{n}^{P}$ over the standard ones $h_{n}^{T}$ and shows the practical sufficiency of the presently known $v^{5}$-accurate post-Newtonian results, we still think that it is an important (and challenging) task to improve the (finite mass) post-Newtonian results. Of particular importance would be the computation [25] of the $v^{6}$-accurate (equations of motion and) energy function in confirming and improving our estimate below of the location of the last stable orbit for $\eta \neq 0$. Our calculations also suggest that knowing $E$ and $F$ to $v^{6}$ would further improve the effectualness (maximized overlap larger than $98 \%$ ) and, more importantly, the faithfulness (diagonal overlap larger than 99.5\%) to a level allowing a loss in the number of detectable events smaller than $1 \%$ and significantly smaller biases (smaller than $0.5 \%$ ) in the parameter estimations than the present $O\left(v^{5}\right)$ results (about $1-5 \%)$.

The rest of this paper is organized as follows: In Sec. II we briefly discuss the phasing of restricted post-Newtonian gravitational wave forms, wherein corrections are only included to the phase of the wave form and not to the amplitude, indicating the way in which energy and flux functions enter the phasing formula. Various forms of energy and flux functions are introduced in Secs. III and IV, respectively, and their performance compared. The ambiguity function, which is the overlap integral of two wave forms as a function of their parameters, is discussed in Sec. V and some details of its computation by a numerical fast Fourier transform are given. In Sec. VI we present the results of our computations in the test mass case while in Sec. VII we investigate the robustness of these test mass results as completely as possible. Section VIII contains our summary and concluding remarks. The paper concludes with two appendixes. In Appendix A we discuss the Padé approximants, their relevant useful properties, and list some useful formulas used in the computations. In Appendix B we discuss carefully the issue of optimizing over the phases and provide a clear geometrical picture to implement the procedure.

\section{PHASING FORMULA}

To get an accurate expression for the evolving wave form $h_{i j}(t)$ emitted by an inspiraling compact binary one needs, in principle, to solve two interconnected problems: (i) One must work out (taking into account propagation and nonlinear effects) the way the material source generates a gravitational wave, and (ii) one must simultaneously work out the evolution of the source (taking into account radiationreaction effects). The first problem, which in a sense deals mainly with the (tensorial) amplitude of the gravitational signal, is presently solved to order $v^{5}[6-10]$. Such an approximation on the instantaneous amplitude $h_{i j}$ seems quite sufficient in view of the expected sensitivity of the LIGO-VIRGO network. On the other hand, the second problem, which determines the evolution of the phase of the gravitational signal, is crucial for a successful detection. For simplicity, we shall work here within the "restricted wave form" approximation [26]; i.e. we shall focus on the main Fourier component of the signal, schematically $h(t)=a^{\mathrm{GW}}(t) \cos \phi^{\mathrm{GW}}(t)$, where the gravitational wave phase $\phi^{\mathrm{GW}}$ is essentially, in the case of a circular binary, twice the orbital phase $\Phi: \quad \phi^{\mathrm{GW}}(t)=2 \Phi(t)$.

We find it conceptually useful to note the analogy between the radio-wave observation of binary pulsars and the gravitational-wave observation of a compact binary. Highprecision observations of binary pulsars make a crucial use of an accurate "timing formula" [27]

$$
\phi_{n}^{\mathrm{PSR}}=F\left[t_{n} ; p_{i}\right],
$$

linking the rotational phase of the spinning pulsar (stroboscopically observed when $\phi_{n}^{\mathrm{PSR}}=2 \pi n$ with $n \in \mathcal{N}$ ) to the time of arrival $t_{n}$ on Earth of an electromagnetic pulse and to some parameters $p_{i}$. Similarly, precise observations of an inspiraling compact binary will need an accurate "phasing formula," i.e. an accurate mathematical model of the continuous evolution of the gravitational wave phase

$$
\phi^{\mathrm{GW}}=2 \Phi=F\left[t ; p_{i}\right],
$$

involving a set of parameters $\left\{p_{i}\right\}$ carrying information about the emitting binary system (such as the two masses $m_{1}$ and $\left.m_{2}\right)$.

Heuristically relying on a standard energy-balance argument, the time evolution of the orbital phase $\Phi$ is determined by two functions: an energy function $E(v)$ and a flux function $F(v)$. Here the argument $v$ is defined by Eq. (1.1) which can be rewritten in terms of the instantaneous orbital angular frequency $\Omega$ :

$$
v \equiv(m \Omega)^{1 / 3} \equiv x^{1 / 2}
$$

(as above $m \equiv m_{1}+m_{2}$ denotes the total mass of the binary). The (dimensionless) energy function $E$ is defined by

$$
E_{\mathrm{tot}}=m(1+E)
$$

where $E_{\text {tot }}$ denotes the total relativistic energy (Bondi mass) of the binary system. The flux function $F(v)$ denotes the gravitational luminosity of the system [at the retarded instant where its angular velocity $\Omega$ is given by Eq. (2.3)]. Note that the three quantities $v, E$ and $F$ are invariantly defined (as 
global quantities in the instantaneous center of mass frame), so that the two functions $E(v), F(v)$ are coordinateindependent constructs. Denoting as above the symmetric mass ratio by $\eta \equiv m_{1} m_{2} /\left(m_{1}+m_{2}\right)^{2}$, the energy balance equation $d E_{\text {tot }} / d t=-F$ gives the following parametric representation of the phasing formula, Eq. (2.2) (written here for the orbital phase):

$$
\begin{gathered}
t(v)=t_{c}+m \int_{v}^{v_{\text {lso }}} d v \frac{E^{\prime}(v)}{F(v)}, \\
\Phi(v)=\Phi_{c}+\int_{v}^{v_{\text {lso }}} d v v^{3} \frac{E^{\prime}(v)}{F(v)}
\end{gathered}
$$

where $t_{c}$ and $\Phi_{c}$ are integration constants, and where for lisibility we have not introduced a new name (such as $v^{\prime}$ ) for the dummy integration variable. Note that $E^{\prime}(v)<0, F(v)$ $>0$ so that both $t$ and $\Phi$ increase with $v$. For definiteness, we have written the integrals in Eqs. (2.5), (2.6) in terms of a specific reference velocity, chosen here to be the velocity corresponding to the last stable circular orbit of the binary. Note that the choice of such a reference point is, in fact, entirely arbitrary and a matter of convention as one introduces the two integration constants $t_{c}$ and $\Phi_{c}$ (which will be optimized later). The choice $v_{\text {ref }}=v_{\text {lso }}$, where $v_{\text {lso }}$ is the velocity at the last stable orbit (lso), is technically and physically natural as it is the value where the integrand vanishes [because of $E^{\prime}(v)$ ]. The definition (and properties) of our approximants do not depend on this choice and the reader is free to use instead his/her favorite reference point. On the other hand, what is not a matter of convention is that, in the absence of information about the coalescence process, we shall also use $v_{\text {lso }}$ to define the time when the inspiral wave form shuts off.

The numerical value of $v_{\text {lso }}$ in the case of a test mass orbiting a black hole (i.e. the limiting case $\eta \rightarrow 0$ ) is $1 / \sqrt{6}$. In the case of binaries of comparable masses $(\eta \neq 0) v_{\text {lso }}$ is the value of $v$ where $E^{\prime}(v)$ vanishes. We will discuss below ways of estimating $v_{\text {lso }}(\eta)$. Knowledge of $v_{\text {lso }}$ (considered now has a physical quantity affecting the signal and not as a simple reference point) is important in gleaning astrophysical information since the inspiral wave form would shut off at that point and the coalescence wave form, whose shape depends on equation of state of stars, etc., would begin. One of the questions we address below is whether (as had been suggested [13]) knowledge of $v_{\text {lso }}(\eta)$ is crucial for getting accurate inspiraling wave templates.

To warm up, let us recall that in the "Newtonian" approximation (i.e. when using the quadrupole formula for the gravitational wave emission) one has

$$
E_{N}(v)=-\frac{1}{2} \eta v^{2}, \quad F_{N}=\frac{32}{5} \eta^{2} v^{10}
$$

so that the above formulas reduce (after redefining the constants of integration or, equivalently, formally setting $v_{\text {lso }}$ $=\infty)$ to

$$
t=t_{c}-\frac{5}{256} m \eta^{-1} v^{-8}, \quad \Phi=\Phi_{c}-\frac{1}{32} \eta^{-1} v^{-5}
$$

The explicit Newtonian phasing formula is obtained by eliminating $v$ and given by

$$
\Phi_{N}(t)=\Phi_{c}-\left(\frac{t_{c}-t}{5 \tau}\right)^{5 / 8}
$$

where $\tau \equiv \eta^{3 / 5} m$ ("chirp time scale"').

The corresponding Newtonian gravitational wave amplitude is (for some constant $C$ )

$$
a_{N}^{\mathrm{GW}}(v)=C v^{2},
$$

so that the explicit Newtonian templates read

$$
h_{N}(t)=C^{\prime}\left(t_{c}-t\right)^{-1 / 4} \cos \left[2 \Phi_{c}-2\left(\frac{t_{c}-t}{5 \tau}\right)^{5 / 8}\right] \text {. }
$$

The crucial issue for working beyond the Newtonian approximation is the availability of sufficiently accurate representations for the two functions $E^{\prime}(v)$ and $F(v)$. In the astrophysically interesting case of two comparable masses orbiting around each other neither of the functions $E(v)$ or $F(v)$ is known exactly and thus one must rely on a postNewtonian expansion for both these quantities. The question is how accurate should our knowledge of the "energy function" $E(v)$ and the "flux function", $F(v)$ be so that we have only an acceptable reduction in the event rate and a tolerable bias in the estimation of parameters. Given some approximants of the energy and flux functions (as functions of $v$ ), say $E_{A}(v), F_{A}(v)$, and given some fiducial velocity [28] $v_{\text {lso }}^{A}$, we shall define a corresponding approximate template

$$
h^{A}=h^{A}\left(t ; C, t_{c}, \Phi_{c}, m, \eta\right)
$$

by the following parametric representation in terms of $v$ :

$$
\begin{gathered}
h^{A}(v)=C v^{2} \cos 2 \Phi_{A}(v), \\
t(v)=t_{c}+m \int_{v}^{v_{\mathrm{lso}}^{A}} d v \frac{E_{A}^{\prime}(v)}{F_{A}(v)}, \\
\Phi_{A}(v)=\Phi_{c}+\int_{v}^{v_{\mathrm{lso}}^{A}} d v v^{3} \frac{E_{A}^{\prime}(v)}{F_{A}(v)} .
\end{gathered}
$$

To compute explicitly $h^{A}(t)$ we numerically invert Eq. (2.14) to get $v=V_{A}(t)$ and substitute the result in the other equations: $h^{A}(t)=C V_{A}^{2}(t) \cos \left[2 \Phi_{A}\left(V_{A}(t)\right)\right]$. Note that we use the Newtonian approximation for the amplitude as a function of $v$. We could use a more refined approximation, such as an effective (main Fourier mode) scalar amplitude $a_{A}^{\mathrm{GW}}(v) \propto \Omega^{-1} F_{A}^{1 / 2} \propto v^{-3} F_{A}^{1 / 2}(v)$. However, our main purpose here being to study the influence of the choice of better approximants to the phase evolution on the quality of the overlaps, it is conceptually cleaner to stick to one common approximation for the amplitude (considered as a function of our principal independent variable $v$ ).

The standard approximants for $E(v)$ and $F(v)$ are simply to use their successive Taylor approximants, Eqs. (1.2), (1.3). Our strategy for constructing new approximants to $E(v)$ and $F(v)$ is going to be two pronged. On the one hand, 
using the knowledge of these functions in the test-mass limit and general theoretical information about their mathematical structure we shall motivate the use of representations of $E(v)$ and $F(v)$ based on other, supposedly more basic energy-type and flux-type functions, say $e(v)$ and $f(v)$. On the other hand, we shall construct Padé-type approximants, say $e_{P_{n}}, f_{P_{n}}$, for the "basic" functions $e(v), f(v)$, instead of straightforward Taylor approximants. We shall then compare the performance of the various phasing formulas defined by inserting in Eqs. (2.13)-(2.15) either the standard $E_{A}^{\text {old }}=E_{T_{n}}, F_{A}^{\text {old }}=F_{T_{n}}$, Eqs. (1.2), (1.3), or the new, two-stage approximants $E_{A}^{\text {new }}=E\left[e_{P_{n}}\right], \quad F_{A}^{\text {new }}=F\left[e_{P_{n}}, f_{P_{n}}\right] . \quad\left[\begin{array}{ll}\text { In } & \text { all }\end{array}\right.$ cases, the approximant of the derivative $E_{A}^{\prime}(v)$ is just $d E_{A}(v) / d v$.]

\section{ENERGY FUNCTION}

Let us motivate the introduction of a new energy function $e(v)$ as a more basic object, hopefully better behaved than the total relativistic mass-energy $E_{\text {tot }}$, Eq. (2.4), of the binary system. For this, let us consider the limit $m_{2} / m_{1} \rightarrow 0$. In this test body limit, i.e. a test particle $m_{2}$ moving in the background of a Schwarzschild black hole of mass $m_{1}$, the total conserved mass-energy of the binary system reads

$$
E_{\text {tot }}=m_{1}+\mathcal{E}_{2}=m_{1}-k_{\mu} p_{2}^{\mu},
$$

where $k_{\mu}$ is the time-translation Killing vector, and $p_{2}^{\mu}$ the 4 -momentum of the test mass. (The quantity $\mathcal{E}_{2} \equiv-k_{\mu} p_{2}^{\mu}$ is the well-known conserved relativistic energy of a test particle moving in a stationary background.) At infinity $k^{\mu}$ $=p_{1}^{\mu} / m_{1}$, so that the formal expression of $E_{\text {tot }}$ is $E_{\mathrm{tot}}=m_{1}$ $-\left(p_{1} \cdot p_{2}\right) / m_{1}$. This expression is clearly very asymmetric in the labels 1 and 2 and has bad analytical properties as a function of $m_{1}$. Both problems are cured by working instead with the standard Mandelstam variable $s=E_{\text {tot }}^{2}=-\left(p_{1}\right.$ $\left.+p_{2}\right)^{2}=m_{1}^{2}+m_{2}^{2}-2\left(p_{1} \cdot p_{2}\right)$. Further, it is known that, in quantum two-body problems, the symmetric quantity

$$
\epsilon \equiv \frac{s-m_{1}^{2}-m_{2}^{2}}{2 m_{1} m_{2}} \equiv \frac{E_{\text {tot }}^{2}-m_{1}^{2}-m_{2}^{2}}{2 m_{1} m_{2}}
$$

is the best energy function to consider when trying to extend one-body-in-external-field results to two-body results [29]. In the limit $m_{2} \ll m_{1}$ the quantity $\boldsymbol{\epsilon}$ reduces simply to $\epsilon$ $=-\left(p_{1} \cdot p_{2}\right) / m_{1} m_{2}=\mathcal{E}_{2} / m_{2}+O(\eta)$.

In the case of a test mass in circular orbit around a Schwarzschild black hole the explicit expression of the quantity $\boldsymbol{\epsilon}$ in terms of the invariant argument $x=v^{2} \equiv(m \Omega)^{2 / 3}$, Eq. (2.3), is

$$
\epsilon=\frac{1-2 x}{\sqrt{1-3 x}}
$$

The explicit test-mass result (3.3) suggests that the (unknown) exact two-body function $\epsilon(x)$ will have also some $\sim\left(x-x_{0}\right)^{-1 / 2}$ singularity in the complex $x$ plane. This led us finally to consider, instead of the function $\epsilon$, its square or, equivalently, the new energy function

$$
e(x) \equiv \epsilon^{2}-1 \equiv\left(\frac{E_{\mathrm{tot}}^{2}-m_{1}^{2}-m_{2}^{2}}{2 m_{1} m_{2}}\right)^{2}-1
$$

Note that we assume here that the total instantaneous relativistic energy of a binary system (in the center of mass frame) can be defined as a time-symmetric functional of positions and velocities [so that $E(v)$ depends on $v$ only through $x$ $\left.\equiv v^{2}\right]$, as the quantity $\widetilde{E}^{\text {even }}$ discussed in Sec. VII of Ref. [30]. It remains, however, unclear whether such a quantity is well defined at very high post-Newtonian orders and whether it is then related to the gravitational wave flux by the standard balance equation.

Summarizing, our proposal is to use as basic (symmetric) energy function the quantity $e(x)$, Eq. (3.4), instead of $E(x) \equiv\left(E_{\text {tot }}-m_{1}-m_{2}\right) /\left(m_{1}+m_{2}\right)$. Given any (approximate or fiducially " "exact") function $e(x)$, we shall then define the corresponding function $E(x)$ (with $x \equiv v^{2}$ ) entering the phasing formulas (2.13)-(2.15) by solving Eq. (3.4) in terms of $E_{\mathrm{tot}} \equiv\left(m_{1}+m_{2}\right)(1+E)$. Explicitly, this gives

$$
E(x)=\{1+2 \eta[\sqrt{1+e(x)}-1]\}^{1 / 2}-1 .
$$

The associated $v$ derivative entering the phasing formula reads

$$
E^{\prime}(v)=\left.2 v \frac{d E(x)}{d x}\right|_{x=v^{2}}=\left.\frac{v \eta}{[1+E(x)] \sqrt{1+e(x)}} \frac{d e(x)}{d x}\right|_{x=v^{2}} .
$$

Having defined our new, basic energy function $e(x)$, it remains to define the approximants of $e(x)$ that we propose to use, when one knows only the Taylor expansion of $E(x)$. For guidance, let us note that by inserting Eq. (3.3) into Eq. (3.4) one gets the following exact expression for the test-mass limit of the function $e(x)$ :

$$
\begin{aligned}
e(x ; \eta=0) & =-x \frac{1-4 x}{1-3 x} \\
& =-x\left(1-x-3 x^{2}-9 x^{3}-\cdots-3^{n-1} x^{n}-\cdots\right) .
\end{aligned}
$$

The generalization of the expansion, Eq. (3.7), to nonzero values of $\eta$ is only known to second post-Newtonian (2PN) accuracy. Using Eq. (4.25) of Ref. [7], that is,

$$
E_{2 \mathrm{PN}}(x)=-\frac{1}{2} \eta x\left[1-\frac{1}{12}(9+\eta) x-\frac{1}{8}\left(27-19 \eta+\frac{\eta^{2}}{3}\right) x^{2}\right]
$$

we compute the $2 \mathrm{PN}$ expansion of the function $e(x)$ for a finite $\eta$ :

$$
e_{2 \mathrm{PN}}(x ; \eta)=-x\left[1-\left(1+\frac{\eta}{3}\right) x-\left(3-\frac{35}{12} \eta\right) x^{2}\right] .
$$

The basic idea behind our proposal is that on the grounds of mathematical continuity [31] between the case $\eta \rightarrow 0$ and the case of finite $\eta$ one can plausibly expect the exact function $e(x)$ to be meromorphically extendable in at least part of the complex plane and to admit a simple pole singularity on the 
TABLE I. Location of the last stable circular orbit determined by the $T$ - and $P$-approximants in the test mass case. The $P$-approximants predict the exact location at orders $v^{4}$ and beyond. At order $v^{2}$ the last stable orbit is not defined by $P$-approximants.

\begin{tabular}{lcc}
\hline \hline$n$ & $x_{\text {lso }}^{T_{n}} / x_{\text {lso }}^{X}$ & $x_{\text {lso }}^{P_{n}} / x_{\text {lso }}^{X}$ \\
\hline & & $x_{\text {lso }}^{X}=0.1667$ \\
& & - \\
6 & 3.0000 & 1.0000 \\
6 & 1.4415 & 1.0000 \\
\hline \hline
\end{tabular}

real axis $\propto\left(x-x_{\text {pole }}\right)^{-1}$ as the nearest singularity in the complex $x$ plane. We do not know the location of this singularity when $\eta \neq 0$, but Padé approximants are excellent tools for giving accurate representations of functions having such pole singularities. For example, if we knew only the $2 \mathrm{PN}$-accurate [i.e. $O\left(v^{4}\right)$ ] expansion of the test-mass energy function (6), namely $e_{2 \mathrm{PN}}(x ; \eta=0)=-x\left(1-x-3 x^{2}\right)$, its corresponding $v^{4}$-accurate diagonal Padé approximant would be uniquely defined (see Appendix A) as

$$
e_{P_{4}}(x ; \eta=0)=-x \frac{1-4 x}{1-3 x},
$$

which coincides with the exact result, Eq. (3.7). Having reconstructed the exact function $e(x)$, we have also reconstructed, using only the information contained in the $2 \mathrm{PN}$ accurate expansion, the existence and location of a last stable orbit. Indeed, using Eqs. (3.6) and (3.10) we find

$$
E_{P_{4}}^{\prime}(v)=-\eta v \frac{1-6 v^{2}}{\left(1-3 v^{2}\right)^{3 / 2}}
$$

which is the exact test mass expression exhibiting a last stable orbit at $v_{\mathrm{lso}}=1 / \sqrt{6}$. In Table I we have compared at different post-Newtonian orders the $x_{1 s 0} \equiv v^{2}$ lso predicted by the standard post-Newtonian series and the Padé approximation to the same. [In the standard post-Newtonian case we defined the lso as the location of the minimum of successive Taylor approximants of the function $E(n)_{\mathrm{v}}$.]

It is important to note that our assumption of structural stability between $e(x ; \eta=0)$ and $e(x ; \eta)$ with $0<\eta \leqslant \frac{1}{4}$ is internally consistent in the sense that the coefficients of $x$ and $x^{2}$ in the square brackets of Eq. (3.9) fractionally change, when $\eta$ is turned on, only by rather small amounts: $\eta / 3 \leqslant \frac{1}{12} \simeq 8.3 \%$ and $35 \eta / 36 \leqslant 35 / 144 \simeq 24.3 \%$, respectively. This contrasts with other attempts to consider $\eta$ as a perturbation parameter, such as Ref. [32]. Indeed, in the quantities considered in the latter work several of the $2 \mathrm{PN}$ terms have coefficients that vary by very large fractional amounts as $\eta$ is turned on: some examples being $12+29 \eta, 2+25 \eta+2 \eta^{2}$, $4+41 \eta+8 \eta^{2}$ in Eqs. (2.2) of the second reference in [32]. Moreover, the fact that many of the coefficients in their Eqs. (2.2) increase when $\eta$ is turned on (like the ones quoted above) is not a good sign for the reliability of their approach as it means, roughly, that the radius of convergence of the particular series they consider tends to decrease as $\eta$ is turned on. We shall attempt below to further test the robustness of our proposal.
In summary, our proposal is the following: Given some usual Taylor approximant to the normal energy function, $E_{T_{2 n}}=-\frac{1}{2} \eta x\left(1+E_{1} x+E_{2} x^{2}+\cdots+E_{n} x^{n}\right)$, one first computes the corresponding Taylor approximant for the $e$ function [33], say

$$
e_{T_{2 n}}=-x \sum_{k=0}^{n} a_{k} x^{k}
$$

in which the only known coefficients are

$$
a_{0}=1, \quad a_{1}=-1-\frac{\eta}{3}, \quad a_{2}=-3+\frac{35 \eta}{12} .
$$

Then, one defines the improved approximant corresponding to Eq. (3.12) by taking the diagonal $\left(P_{m}^{m}\right.$, if $\left.n=2 m\right)$ or subdiagonal $\left(P_{m+1}^{m}\right.$, if $\left.n=2 m+1\right)$ Padé approximant of $-x^{-1} e_{T_{2 n}}(x)$ :

$$
e_{P_{2 n}}(x) \equiv-x P_{m+\epsilon}^{m}\left[\sum_{k=0}^{n} a_{k} x^{k}\right],
$$

where $\epsilon=0$ or 1 depending on whether $n \equiv 2 m+\epsilon$ is even or odd. For completeness, we recall the definition and basic properties of Padé approximants in Appendix A. Let us only mention here that the $P_{m+\epsilon}^{m}$ approximants are conveniently obtained as a continued fraction. For instance, the Padé approximant of the $2 \mathrm{PN}$-approximate $e_{T_{4}}(x)=-x\left(a_{0}+a_{1} x\right.$ $\left.+a_{2} x^{2}\right)$ is

$$
e_{P_{4}}(x)=\frac{-x c_{0}}{1+\frac{c_{1} x}{1+c_{2} x}}=\frac{-c_{0} x\left(1+c_{2} x\right)}{1+\left(c_{1}+c_{2}\right) x} .
$$

By demanding that this agree with $e_{T_{4}}$ to order $v^{4}$ we can relate the $c_{n}$ 's in the above equation to the $a_{n}$ 's in Eq. (3.13):

$$
c_{0}=a_{0}, \quad c_{1}=-\frac{a_{1}}{a_{0}}, \quad \text { and } \quad c_{2}=-\frac{a_{2}}{a_{1}}+\frac{a_{1}}{a_{0}} .
$$

Explicitly, this gives

$$
\begin{gathered}
c_{0}=1, \quad c_{1}=1+\frac{\eta}{3}, \\
c_{2}=-c_{1}-\frac{3-\frac{35}{12} \eta}{1+\frac{1}{3} \eta}=-\frac{4-\frac{9}{4} \eta+\frac{1}{9} \eta^{2}}{1+\frac{1}{3} \eta},
\end{gathered}
$$

so that

$$
e_{P_{4}}(x)=-x \frac{1+\frac{1}{3} \eta-\left(4-\frac{9}{4} \eta+\frac{1}{9} \eta^{2}\right) x}{1+\frac{1}{3} \eta-\left(3-\frac{35}{12} \eta\right) x} .
$$

Given a continued fraction approximant $e_{\mathrm{P}_{n}}(x)$ of the truncated Taylor series $e_{T_{n}}$ of the energy function $e(x)$ the corresponding $E(x)$ and $E^{\prime}(x)$ functions are obtained using 


$$
\begin{gathered}
E_{P_{n}}(x)=\left[1+2 \eta\left(\sqrt{1+e_{P_{n}}(x)}-1\right)\right]^{1 / 2}-1, \\
E_{P_{n}}^{\prime}(v)=\left.2 v \frac{d E_{P_{n}}(x)}{d x}\right|_{x=v^{2}} \\
=\left.\frac{v \eta}{\left[1+E_{P_{n}}(x)\right] \sqrt{1+e_{P_{n}}(x)}} \frac{d e_{P_{n}}(x)}{d x}\right|_{x=v^{2}} .
\end{gathered}
$$

Thus, for instance,

$$
\begin{aligned}
\hat{E}_{P_{4}}^{\prime}(v) & \equiv \frac{-E_{P_{4}}^{\prime}(v)}{\eta v} \\
& =\frac{c_{0}\left[1+2 c_{2} v^{2}+\left(c_{1} c_{2}+c_{2}^{2}\right) v^{4}\right]}{\left[1+\left(c_{1}+c_{2}\right) v^{2}\right]^{2}\left[1+E_{P_{4}}\left(v^{2}\right)\right] \sqrt{1+e_{P_{4}}\left(v^{2}\right)}},
\end{aligned}
$$

where $E_{P_{n}}$ is given by Eq. (3.19). The careted notation introduced on the left-hand side of Eq. (3.21) will again be used below and indicates that one is dividing some function of $v$ by its Newtonian approximation: e.g. $\hat{E}^{\prime}(v)$ $\equiv E^{\prime}(v) / E_{N}^{\prime}(v)$ where, from Eq. (2.7), $E_{N}^{\prime}(v)=-\eta v$.

Having argued that $e_{P_{4}}(x)$, Eq. (3.18), and the corresponding $E_{P_{4}}(x)$ defined by Eq. (3.19) are better estimates of the finite-mass energy functions than their straightforward post-Newtonian approximations, Eqs. (3.8), (3.9), we can use our results so far to estimate both the location of the last unstable circular orbit (light ring) and that of the last stable circular orbit. The functions $e_{P_{4}}(v), \hat{E}_{P_{4}}^{\prime}(v)$ are plotted in Fig. 2 together with $e_{T_{4}}(v)$ and $\hat{E}_{T_{4}}^{\prime}(v)$, both sets for $\eta$ $=1 / 4$, and compared with the exact functions $e(v)$ and $\hat{E}^{\prime}(v)$ in the $\eta=0$ (i.e. test mass) case. We see that the $\eta$ $=1 / 4 P$ - and $T$-approximants are smooth deformations of their test-mass limits. Note that the variable $x \equiv v^{2}$ is, in the limit $\eta \rightarrow 0$, equal to $m / r$ in Schwarzschild coordinates and can be used as a smooth radial coordinate. If we wished, we could also introduce the function $J_{\text {tot }}(x)$, giving the $x$ variation of the total angular momentum. It is indeed related to the total energy $E_{\text {tot }}(x)$ by the general identity (for circular orbits) $d E_{\text {tot }}=\Omega d J_{\text {tot }}$ where the circular frequency is given by $m \Omega=v^{3}=x^{3 / 2}$. The consideration (even without knowing its precise analytical form) of the effective potential for general (noncircular) orbits $E_{\text {tot }}=E_{\text {tot }}\left(r, J_{\text {tot }}\right)$ in terms of any smooth radial-type variable $r$ measuring the distance between the two bodies allows one to see (by smooth deformation from the $\eta=0$ case) that the minimum of $E_{\text {tot }}(x)$ [which necessarily coincides with the minimum of $J_{\text {tot }}(x)$ ] defines the last stable circular orbit. Indeed, it is the confluence of the one-parameter sequence of minima of $E_{\text {tot }}\left(r, J_{\text {tot }}\right)$ considered as a function of $r$ for fixed $J_{\text {tot }}$ (stable circular orbits) with the one-parameter sequence of maxima of $E_{\mathrm{tot}}\left(r, J_{\text {tot }}\right)$ (unstable circular orbits). Note also, from Eq. (3.20), that the last stable orbit [minimum of $E(x)$ ] necessarily coincides with the minimum of the function $e(x)$. As for the last unstable circular orbit it is clearly defined by the square-root
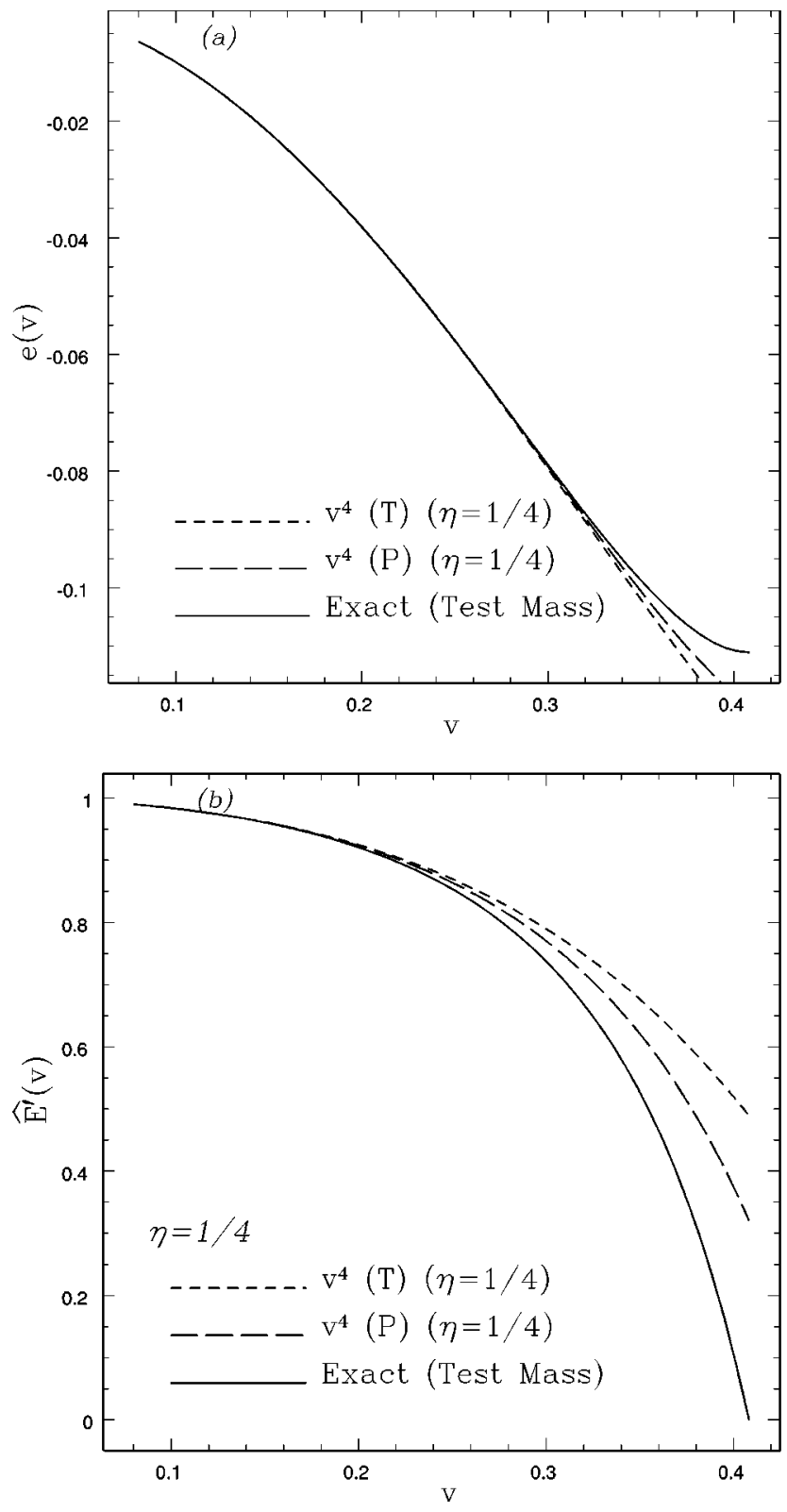

FIG. 2. Exact energy functions (a) $e(v)$ and (b) the Newtonnormalized $\hat{E}^{\prime}(v)$, in the test mass case and $T$ - and $P$-approximants in the comparable mass (with $\eta=1 / 4$ ) case. Note that the comparable mass cases $T$-approximant and $P$-approximant, are smooth deformations of the test mass function.

singularity $\propto\left(x-x_{\text {pole }}\right)^{-1 / 2}$ of $E(x)$, corresponding to a simple pole $\left(x-x_{\text {pole }}\right)^{-1}$ in $e(x)$. Applying these general considerations to our specific 2PN-Padé proposal (3.18) one easily finds that we predict the following "locations" (in the invariant $x$ variable) for both the light ring (corresponding to $r=3 m$ for a test mass around a Schwarzschild black hole),

$$
x_{\text {light ring }}^{P_{4}}(\eta)=x_{\text {pole }}^{P_{4}}(\eta)=\frac{1}{3} \frac{\left(1+\frac{1}{3} \eta\right)}{\left(1-\frac{35}{36} \eta\right)},
$$

and for the last (circular) stable orbit, 


$$
x_{\mathrm{lso}}^{P_{4}}(\eta)=\frac{1}{6} \frac{\left(1+\frac{1}{3} \eta\right)}{\left(1-\frac{35}{36} \eta\right)}\left[2-\frac{\left(1+\frac{1}{3} \eta\right)}{\sqrt{1-\frac{9}{16} \eta+\frac{1}{36} \eta^{2}}}\right] .
$$

We recall that $x$ is invariantly defined in terms of the orbital circular frequency $\Omega=2 \pi f^{\text {orb }}$ through $x=(m \Omega)^{2 / 3}$, so that the gravitational wave frequency (twice the orbital frequency) reads

$$
f^{\mathrm{GW}}=2 f^{\mathrm{orb}}=\frac{x^{3 / 2}}{\pi m}=4397.2(6 x)^{3 / 2} \frac{m_{\odot}}{m} \mathrm{~Hz} .
$$

In the equal mass case ( $\eta=1 / 4)$ Eqs. (3.22), (3.23) yield $3 x_{\text {pole }}^{P_{4}}\left(\frac{1}{4}\right)=156 / 109 \simeq 1.4312,6 x_{1 \text { so }}^{P_{4}}\left(\frac{1}{4}\right) \simeq 1.1916$, and therefore $f_{\text {lso }}^{\mathrm{GW}}=2 f_{\mathrm{lso}}^{\mathrm{orb}}=5719.4\left(m_{\odot} / \mathrm{m}\right) \mathrm{Hz}$. In particular, for a $\left(1.4 m_{\odot}, 1.4 m_{\odot}\right)$ neutron star system, we predict $f_{\text {lso }}^{\mathrm{GW}}$ $=2 f_{\mathrm{lso}}^{\mathrm{orb}}=2042.6 \mathrm{~Hz}$, and for a $\left(10 M_{\odot}, 10 M_{\odot}\right)$ black hole system we predict $f_{\text {lso }}=2 f_{\text {orb }}=286.0 \mathrm{~Hz}$. Note that our estimate of the (invariant) location of the last stable orbit is significantly different from that of Ref. [32], which estimates, for instance $f_{\text {lso }}^{\mathrm{GW}}=2 f_{\mathrm{lso}}^{\mathrm{orb}}=1420 \mathrm{~Hz}$ for a $\left(1.4 m_{\odot}, 1.4 m_{\odot}\right)$ system. [Actually, we read in the figures of Ref. [32] a value $\left(m f_{\mathrm{lso}}^{\text {orb }}\right){ }^{\mathrm{KWW}} \simeq 0.00963$ which corresponds to $6 x_{\mathrm{lso}}^{\mathrm{KWW}} \simeq 0.925$ and $f_{\text {lso }}^{\text {orb }} \simeq 698 \mathrm{~Hz}$ (instead of $710 \mathrm{~Hz}$ marked on their figures) for the $\left(1.4 m_{\odot}, 1.4 m_{\odot}\right)$ case.] Qualitatively our $\eta$ dependence is different because we find that $x_{\mathrm{lso}}^{P_{4}}(\eta)$ increases with $\eta\left[6 x_{\mathrm{lso}}^{P_{4}}(\eta)>1\right.$ and increasing] while Ref. [32] estimates a $6 x_{\mathrm{lso}}^{\mathrm{KWW}}(\eta)<1$, decreasing with $\eta$. This is an important physical difference as it means, if we are right, that binary systems of comparable masses can get closer, orbit faster and emit more gravitational waves before plunging in than estimated in Ref. [32]. As said above, we think that the "hybrid" approximation used in Ref. [32] is not reliable, notably because of the strong $\eta$ dependence (and consequent increase) of the coefficients in their expansion (see also the related criticism of Ref. [34]). We think that our approach [in which the expansion coefficients to $e(x)$ are less strongly modified by $\eta$ and where the crucial coefficient $a_{2}$ decreases with $\eta$ which means a larger radius of convergence] is more likely to indicate the correct trend. We have tried in several ways to test the robustness of our conclusions under the addition of higher post-Newtonian corrections to Eq. (3.9). We think, however, that such attempts are not really conclusive because one does not know in advance what is the "plausible" range of values of 3PN and higher $\eta$-dependent corrections. [We note in this respect that the range considered in Ref. [32], $\left|\alpha_{i}\right|_{\max }=\left|\beta_{i}\right|_{\max }=10$, is clearly too small as it means, for instance, a fractional change in the coefficient of $(\mathrm{m} / \mathrm{r})^{3}$ when $\eta$ changes from 0 to $1 / 4$ of $\eta\left|\alpha_{3}\right| / 16<16 \%$, while the known fractional change in the coefficient of $(m / r)^{2}$ is already $\eta 29 / 12>60 \%$.] In fact, the relative change [the ratio $a_{k}(1 / 4) / a_{k}(0)$ when $\eta$ changes from 0 to 1/4] of the successive coefficients in any power series, such as the $a_{k}(\eta)$ in Eq. (3.12), is expected to increase (or decrease) exponentially with the order $k$ due to an $\eta$-dependent shift of the convergence radius. For instance, in our case if we write the 3PN coefficient as $a_{3}(\eta)$ $=-9\left(1+\kappa_{3} \eta\right)$ to model the 3PN $\eta$ dependence it is not meaningful to consider a priori that $\kappa_{3}$ can take any values in the range $\pm \kappa_{2} \simeq \pm 1$ [where we introduced $a_{2}(\eta)$ $=-3\left(1+\kappa_{2} \eta\right)$ with $\left.\kappa_{2}=-35 / 36\right]$. As the negative value of $\kappa_{2}$ has indicated an increase of the radius of convergence with $\eta\left[x_{\text {pole }}^{P_{4}}(\eta)=a_{1}(\eta) / a_{2}(\eta)=\frac{1}{3}\left(1+\kappa_{1} \eta\right) /\left(1+\kappa_{2} \eta\right)\right.$ with $\kappa_{1}=1 / 3$ ] we would rather expect a value of $\kappa_{3}$ such that $a_{3} / a_{2} \sim a_{2} / a_{1}$, i.e. $1+\kappa_{3} \eta \sim\left(1+\kappa_{2} \eta\right)^{2} /\left(1+\kappa_{1} \eta\right)$ so that $\kappa_{3} \sim-1.9$. A value of $\kappa_{3}$ very different from this estimate [i.e. a value of $a_{3}(1 / 4)$ very different from -4.8] would mean that the coefficient $a_{2}(1 / 4)$ was accidentally smaller than normal [in which case our estimates (3.22), (3.23) would not be reliable]. In conclusion, we think that, given the presently available information, our estimates are more internally consistent than previous ones (which include the relevant works quoted in Ref. [32]), but that, if $a_{2}(\eta)$ is only "accidentally" decreased by turning on $\eta$, they might be off the mark. It will be possible to make more precise statements on the reliability of Eq. (3.23) only when the 3PN equations of motion of a binary system are derived (or when numerical calculations can reliably locate the last stable orbit). Anyway, we shall see that a knowledge of the lso is not so crucial for extracting the inspiral wave form. [We shall notwithstanding test below the robustness of our overall approach under possible uncertainties in the locations of $x_{\text {pole }}(\eta)$ and $x_{\text {lso }}(\eta)$.] This is because, (a) interferometer noise rises quadratically beyond a certain frequency; consequently the noise level is pretty high before light binaries, such as neutron-star-neutron-star (NS-NS) and NS-blackhole $(\mathrm{BH})$, reach the LSO; only in the case of more massive binaries consisting of black holes and/or supermassive stars with total mass in excess of $25 M_{\odot}$, in the case of initial LIGO, and $60 M_{\odot}$, in the case of advanced LIGO, will the frequency at the lso be in a region where the detector noise is low. In such cases it is important to know the location of the lso accurately because it helps in appropriately truncating the inspiral wave form in search templates so that it would not produce anticorrelation with the coalescence wave form which is itself not known, as of now, to any accuracy. In the case of lighter mass binaries what is really needed is that the approximate energy function should match the exact one at frequencies where the detector noise is the least. This is also true for the flux function as we shall see in the next section.

\section{FLUX FUNCTION}

Contrary to the case of the energy function where we could draw on a lot of theoretical information, we have less general a priori information on the structure of the flux function $F(v)$. The exact gravitational wave luminosity $F$ is not known analytically. It has, however, been computed numerically with good accuracy in the test particle limit $[11,13]$ and we shall use this in our study. In the test particle limit the flux is also known analytically to a high order in perturbation theory; to order $v^{11}[11]$ we have

$$
\begin{aligned}
F(v ; \eta=0) & =\frac{32}{5} \eta^{2} v^{10}\left[\sum_{k=0}^{11} A_{k} v^{k}+\left(B_{6} v^{6}+B_{8} v^{8}+B_{9} v^{9}\right.\right. \\
& \left.\left.+B_{10} v^{10}+B_{11} v^{11}\right) \ln v\right]
\end{aligned}
$$


where the various coefficients $A_{k}$ and $B_{k}$ can be read off from [11]:

$$
\begin{gathered}
A_{0}=1, \\
A_{1}=0, \\
A_{2}=-3.711309523809524, \\
A_{3}=12.56637061435917, \\
A_{4}=-4.928461199294533, \\
A_{5}=-38.29283545469344, \\
A_{6}=115.7317166756113, \\
A_{7}=-101.5095959597416, \\
A_{8}=-117.5043907226773, \\
A_{9}=719.1283422334299, \\
A_{10}=-1216.906991317042, \\
A_{11}=958.934970119567, \\
B_{6}=-16.3047619047619, \\
B_{8}=52.74308390022676, \\
B_{9}=-204.8916808741229, \\
B_{10}=116.6398765941094, \\
B_{11}=473.6244781742307,
\end{gathered}
$$

By contrast, in the comparable masses case only the first five Taylor approximants of $F(v ; \eta)$ are known [6-10]. Explicitly, $B_{k}(\eta)=0(k \leqslant 5)$ and

$$
\begin{gathered}
A_{0}(\eta)=1, \\
A_{1}(\eta)=0, \\
A_{2}(\eta)=-\frac{1247}{336}-\frac{35}{12} \eta, \\
A_{3}(\eta)=4 \pi, \\
A_{4}(\eta)=-\frac{44711}{9072}+\frac{9271}{504} \eta+\frac{65}{18} \eta^{2}, \\
A_{5}(\eta)=-\left(\frac{8191}{672}+\frac{535}{24} \eta\right) \pi .
\end{gathered}
$$

There is, however, a bit of general information about the function $F(v)$ which can be used to motivate the consideration of a transformed flux function, say $f(v)$, as a better behaved object. Indeed, as pointed out in Ref. [2], the function $F(v ; \eta=0)$ has a simple pole at the light ring $(r=3 m$, i.e. $\left.x \equiv v^{2}=\frac{1}{3}\right)$. The origin of this pole is simple to understand physically in a flat spacetime analogue. [It is seen from
Refs. [1] and [2] that the curved-spacetime effects (metric coefficients, Green function) do not play an essential role and that the origin of the pole can be directly seen in the source terms, Eqs. (2.14) of Ref. [1].] Let us consider two (for simplicity identical) mass points, linked by a relativistic (Nambu-Goto) string, orbiting around each other on a circle (the string tension $T$ providing the centripetal force opposing centrifugal effects). One can easily find the exact solution of this problem and then estimate the linearized gravitational waves emitted by the system [35]. Let us keep fixed the rest masses $m_{1}=m_{2}=m / 2$ and the radius of the orbit $R$ and increase the tension $T$ so that the particles' velocities $v$ tend to the velocity of light. In this limit, one finds that $R T \sim p$ $=m v / \sqrt{1-v^{2} / c^{2}}$ and that the gravitational wave amplitude $h \propto R T+p \sim p$. By taking a time derivative and squaring one sees that, as $v \rightarrow c$, the gravitational flux $F \sim \Omega^{2} h^{2} \propto p^{2}$ tends to infinity like $\left(1-v^{2} / c^{2}\right)^{-1}$. This shows that the finding of Refs. [1,2] is quite general and that, in particular, it is very plausible that a binary system of comparable masses will have a simple pole in $F(v)$ when the bodies tend to the light ring orbit. We have seen above that the light ring orbit corresponds to a simple pole $x_{\text {pole }}(\eta)$ in the new energy function $e(x ; \eta)$. Let us define the corresponding (invariant) "velocity" $v_{\text {pole }}(\eta) \equiv \sqrt{x_{\text {pole }}(\eta)}$. This motivates the introduction of the following "factored" flux function:

$$
f(v ; \eta) \equiv\left(1-\frac{v}{v_{\text {pole }}(\eta)}\right) F(v ; \eta)
$$

Note that multiplying by $1-v / v_{\text {pole }}$ rather than 1 $-\left(v / v_{\text {pole }}\right)^{2}$ has the advantage of regularizing the structure of the Taylor series of $f(v)$ in introducing a term linear in $v$ [which is absent in Eq. (4.1)]. Two further tricks will allow us to construct well-converging approximants to $f(v)$. First, it is clear (if we think of $v$ as having the dimension of a velocity) that one should normalize the velocity $v$ entering the logarithms in Eq. (4.1) to some relevant velocity scale $v_{0}$. In the absence of further information the choice $v_{0}$ $=v_{\text {lso }}(\eta)$ seems justified (the other basic choice $v_{0}=v_{\text {pole }}$ is numerically less desirable as $v$ will never exceed $v_{\text {lso }}$ and we wish to minimize the effect of the logarithmic terms). A second idea, to reduce the problem to a series amenable to Padéing, is to factorize the logarithms by writing the $f$ function in the form

$$
\begin{aligned}
f(v ; \eta)= & \frac{32}{5} \eta^{2} v^{10}\left[1+\ln \frac{v}{v_{\mathrm{lso}}(\eta)}\left(\sum_{k} \ell_{k} v^{k}\right)+\cdots\right] \\
& \times\left[\sum_{k} f_{k} v^{k}\right] .
\end{aligned}
$$

The ellipses in Eq. (4.5) is meant to represent possible higher powers of $\ln \left(v / v_{\text {lso }}\right)$. (Such terms do not show up at order $v^{11}$ when $\eta=0$ and will be also of no concern when considering the $\eta \neq 0$ results at order $v^{6}$.) The coefficients $f_{k}$ are functions of $v_{\text {lso }}$ in general [36].

Finally, we define our approximants to the factored flux function $f(v)$ as 


$$
\begin{aligned}
f_{P_{n}}(v ; \eta) \equiv & \frac{32}{5} \eta^{2} v^{10}\left[1+\ln \frac{v}{v_{\text {lso }}^{P_{n}}(\eta)}\left(\sum_{k \geqslant 6} \ell_{k} v^{k}\right)\right. \\
& +\cdots] P_{m+\epsilon}^{m}\left[\sum_{k=0}^{n} f_{k} v^{k}\right],
\end{aligned}
$$

where $v_{\mathrm{lso}}^{P_{n}}(\eta)$ denotes the lso velocity $\left(\equiv \sqrt{x_{\mathrm{lso}}}\right)$ for the $v^{n}$-accurate Padé approximant of $e(x)$, and where $P_{m+\epsilon}^{m}$ denotes as before a diagonal or subdiagonal Padé approximant with $n \equiv 2 m+\epsilon, \epsilon=0$ or 1 . The corresponding approximant of the flux $F(v)$ is then defined as

$$
F_{P_{n}}(v ; \eta) \equiv\left(1-\frac{v}{v_{\text {pole }}^{P_{n}}(\eta)}\right)^{-1} f_{P_{n}}(v ; \eta),
$$

where $v_{\text {pole }}^{P_{n}}(\eta)$ denotes the pole velocity defined by the $v^{n}$ Padé approximant of $e(x)$. For instance, from Eq. (3.22)

$$
v_{\text {pole }}^{P_{4}}(\eta)=\frac{1}{\sqrt{3}}\left(\frac{1+\frac{1}{3} \eta}{1-\frac{35}{36} \eta}\right)^{1 / 2} .
$$

Let us now see what this definition gives in practice. In terms of the original expansion coefficients of $F(v), A_{k}$ and $B_{k}$ (considered for any $\eta$ ) and of the fiducial velocity $v_{0}$ $\equiv v_{\text {lso }}$, the coefficients appearing in the definition (4.6) read

$$
\begin{gathered}
\ell_{6}=B_{6}, \\
\ell_{7}=0, \\
\ell_{8}=B_{8}-A_{2} B_{6}, \\
\ell_{9}=B_{9}-A_{3} B_{6}, \\
\ell_{10}=B_{10}-A_{2} \ell_{8}-A_{4} B_{6}, \\
\ell_{11}=B_{11}-A_{2} \ell_{9}-A_{3} \ell_{8}-A_{5} B_{6} .
\end{gathered}
$$

We find (remarkably?) that in the test particle limit the $O\left(v^{9}\right)$ logarithmic term vanishes identically: $\ell_{9}(\eta=0) \equiv 0$. The other coefficients are numerically $\left(\eta=0, v_{\text {pole }}=1 / \sqrt{3}\right.$, $\left.v_{0}=v_{\mathrm{lso}}=1 / \sqrt{6}\right), \quad f_{0}=1, \quad f_{1}=-1.7320508075689, \quad f_{2}=$ $-3.7113095238095, \quad f_{3}=18.994547272212, \quad f_{4}=$ $-26.694053570105, \quad f_{5}=-29.756490254383, \quad f_{6}$ $=196.66395901720, \quad f_{7}=-327.26305863109, \quad f_{8}$ $=11.063926928123, \quad f_{9}=1188.0521512280, \quad f_{10}=$ -2884.9014287843 , and $f_{11}=2823.3603070298$. As for the $\log$ factor in Eq. (4.6) we find that when it is not identically 1 (i.e. when $n \geqslant 6$ ) it is always smaller than about 1.005 for $v \leqslant v_{\text {lso }} \simeq 0.40825$ and much closer to 1 when $v \leqq 0.2$. Although it is unpleasant to have logarithms mixing with powers, they do not seem to introduce, in the present case (after normalization to $v_{\text {lso }}$ and factorization), a serious obstacle to constructing good approximants to $f(v)$.

Our primary aim in this work is to compare and contrast the convergence properties of the standard ("Taylor") approximants to the phasing formula and its building blocks
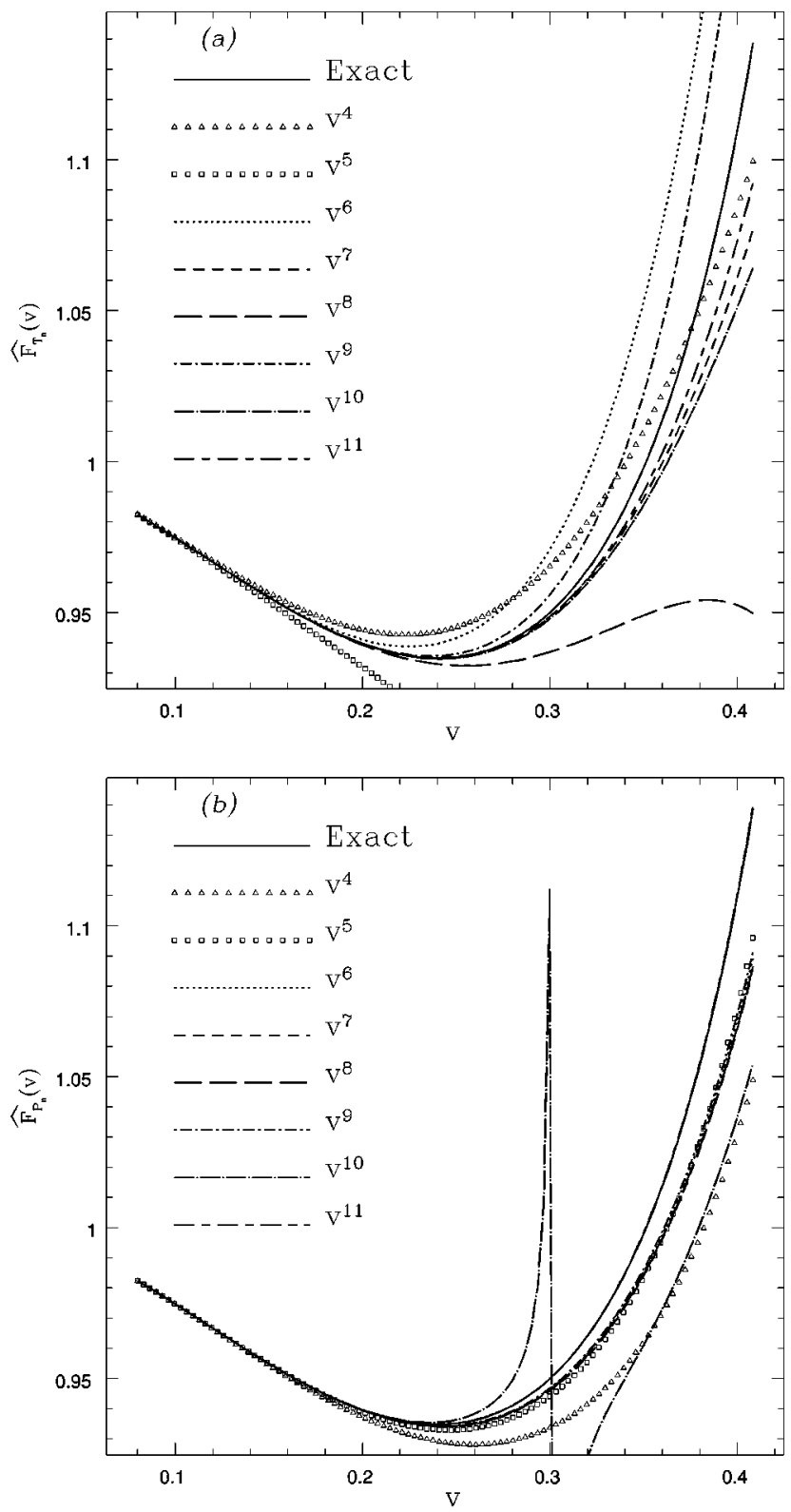

FIG. 3. Newton-normalized gravitational wave luminosity in the test particle limit: (a) $T$-approximants and (b) $P$-approximants.

$E(v)$ and $F(v)$ with the new approximants defined above (with their two-stage construction $E\left[e_{P}\right]$ and $F\left[f_{P}\right]$ ). Let us first discuss the case of the flux function which can be studied in detail in the limiting case $\eta \rightarrow 0$. Indeed, in this case one knows both the "exact" (numerical) flux function [13], say $F_{X}(v)$ and its post-Newtonian expansion up to order $v^{11}$ [11]. We can then compare directly the approach toward $F_{X}(v)$, on the one hand, of the successive standard Taylor approximants $F_{T_{n}}(v ; \eta=0)$ [obtained by keeping only the $A_{k}$ and $B_{k}$ with $k \leqslant n$ in Eq. (4.1)] and, on the other hand, of the new approximants $F_{P_{n}}(v ; \eta=0)$ defined by Eqs. (4.6). This comparison of convergence is illustrated in Fig. 3. We have plotted there, for convenience, the "Newtonnormalized" flux functions

$$
\hat{F}_{A}(v) \equiv \frac{F_{A}(v)}{F_{N}(v)} \equiv \frac{5}{32} \eta^{-2} v^{-10} F_{A}(v) .
$$


It is clear that the $P$-approximants converge to the exact values much faster than the Taylor ones. The monotonicity of the convergence of the $P$-approximants is also striking. However, the $P$-approximants of the flux at certain orders (notably $v^{7}$ and $v^{10}$ ) exhibit poles that happen to lie in the region of integration: $v_{\text {low }}<v<v_{\text {lso }}$. Such $P$-approximants are obviously a bad choice for the construction of templates. Nevertheless, this does not mean that one cannot construct $P$-approximants at that order at all. Recall that in this study we have only considered diagonal and subdiagonal Padé approximants of type $P_{m}^{m}$ and $P_{m+\epsilon}^{m}$, respectively. It is perfectly legitimate to employ other types of Padé approximants and in particular the superdiagonal Padé approximant of type $P_{m}^{m+\epsilon}$. For instance, there is a pole in the region of interest in the $P_{4}^{3}$-approximant of the flux while it turns out that the $P_{3}^{4}$-approximant (which is the one we have used in this work instead of $P_{4}^{3}$ ) does not have a pole in the region of interest. Thus, if one wishes, one may trade off a spurious zero, in the region of interest, in the denominator of the function with a zero of the numerator, thereby removing the troublesome pole (see Appendix A for how this may be accomplished via some simple properties of the Padé approximants). For completeness we exhibit in Fig. 4 the successive $P$-approximants to the factored flux function $f(v ; \eta=0)$.

The other building blocks of the phasing formula, Eqs. (2.13)-(2.15), are the approximants to the function $E^{\prime}(v)$ $=d E(v) / d v$. As we have constructed $E_{P_{n}}(v)$ so that it coincides for $n \geqslant 4$ with the exact $E_{X}(v)$ in the case $\eta=0$ it would not be fair to compare it to the straightforward $E_{T_{n}}(v)$. We need, therefore, to consider the finite mass case $\eta \neq 0$. However, in this case, we only know few PN approximations and we do not know the exact result. We can formally bypass this problem and have a first test of the robustness of our construction by defining the following fiducial "exact' energy function $e_{X}^{\kappa_{0}}(x)$ :

$$
e_{X}^{\kappa_{0}}(x ; \eta) \equiv-x\left[1-\left(1+\frac{\eta}{3}\right) x-\frac{\left(3-\frac{35}{12} \eta\right) x^{2}}{1-3\left(1-\kappa_{0} \eta\right) x}\right] .
$$

The 2PN expansion of $e_{X}^{\kappa_{0}}(x)$ coincides by construction with that of the "real" $e(x ; \eta)$. The parameter $\kappa_{0}$ enters only 3PN and higher order terms. Note that $\kappa_{0}$ parametrizes an infinite number of PN terms in a nonperturbative manner because it determines the location of the pole singularity of $e_{X}^{\kappa_{0}}$, namely

$$
3 x_{\text {pole }}^{\kappa_{0}}=\frac{1}{1-\kappa_{0} \eta} .
$$

If we believe our 2PN Padé estimate (3.22), we would expect that a good estimate of the "real" $\kappa_{0}$ (when considering $\eta$

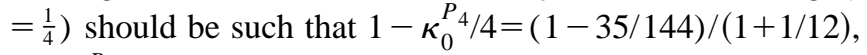
i.e. $\kappa_{0}^{P_{4}}=+47 / 39 \simeq+1.2051$. To test formally the convergence of the sequence of $P$-approximants away from the region where we know by construction that it would converge very fast we shall consider a value of $\kappa_{0}$ substantially different from the Padé-expected one, for instance simply
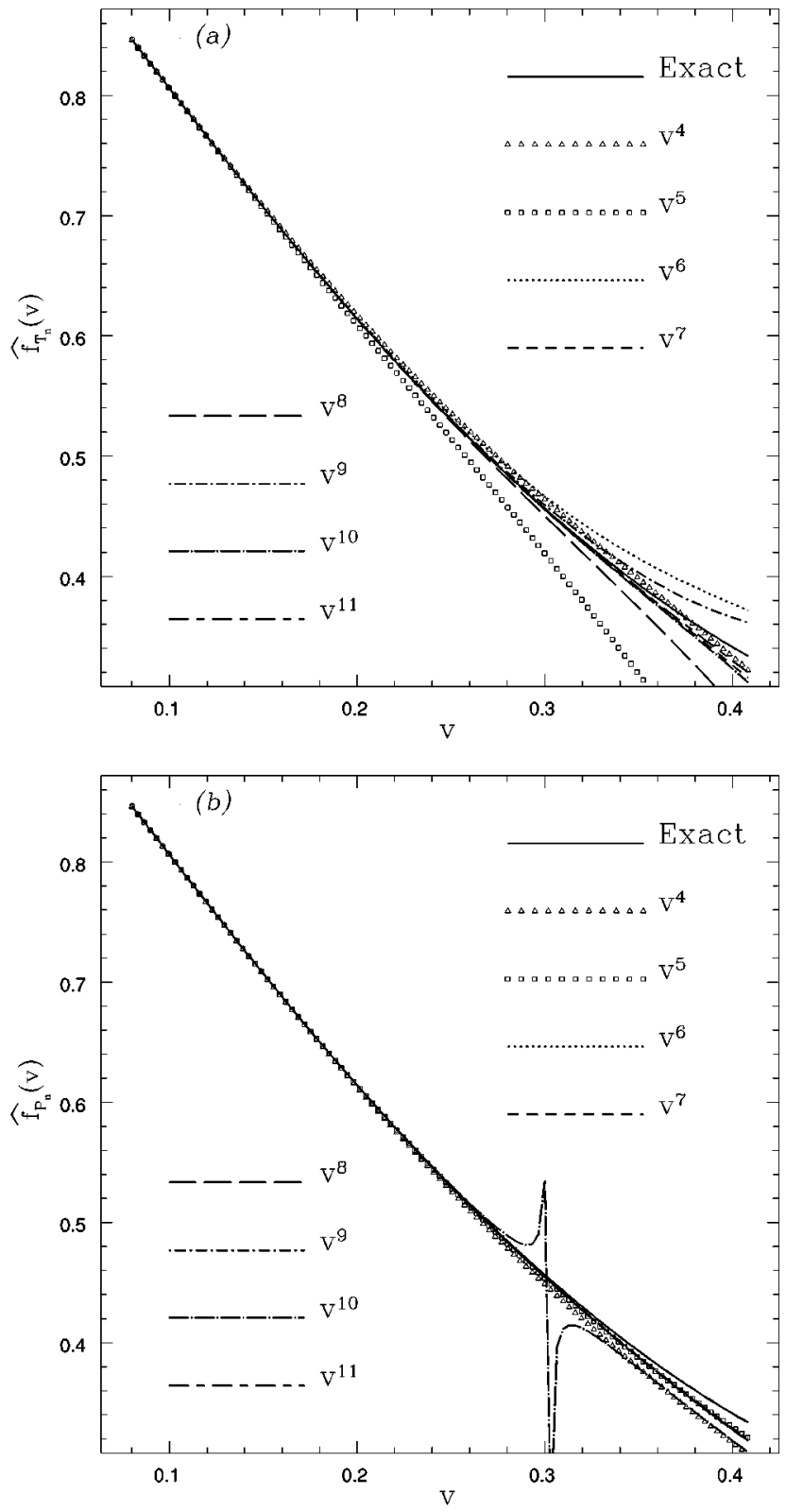

FIG. 4. Newton-normalized factored gravitational wave luminosity in the test particle limit: (a) $T$-approximants and (b) $P$-approximants.

$\kappa_{0}=0$ which says that the "exact" pole stays when $\eta \neq 0$ at the test mass value $3 x=1$ instead of our result above $3 x_{\text {pole }}^{P_{4}}\left(\frac{1}{4}\right) \simeq 1.4312$. Working again with "Newton-normalized" functions, now

$$
\hat{E}_{A}^{\prime}(v) \equiv \frac{E_{A}^{\prime}(v)}{E_{N}^{\prime}(v)} \equiv-\eta^{-1} v^{-1} \frac{d E_{A}(v)}{d v}
$$

we compare in Fig. 5 the convergence of $\hat{E}_{T_{n}}^{\prime}(v)$ and $\hat{E}_{P_{n}}^{\prime}(v)$ toward the fiducial "exact" $e_{X}^{\kappa_{0}}(v)$ defined by Eq. (4.11) for $\eta=\frac{1}{4}, \kappa_{0}=0$. For completeness we exhibit also in Fig. 6 the successive $P$-approximants to the "basic" energy function $e_{X}^{\kappa_{0}}$. 


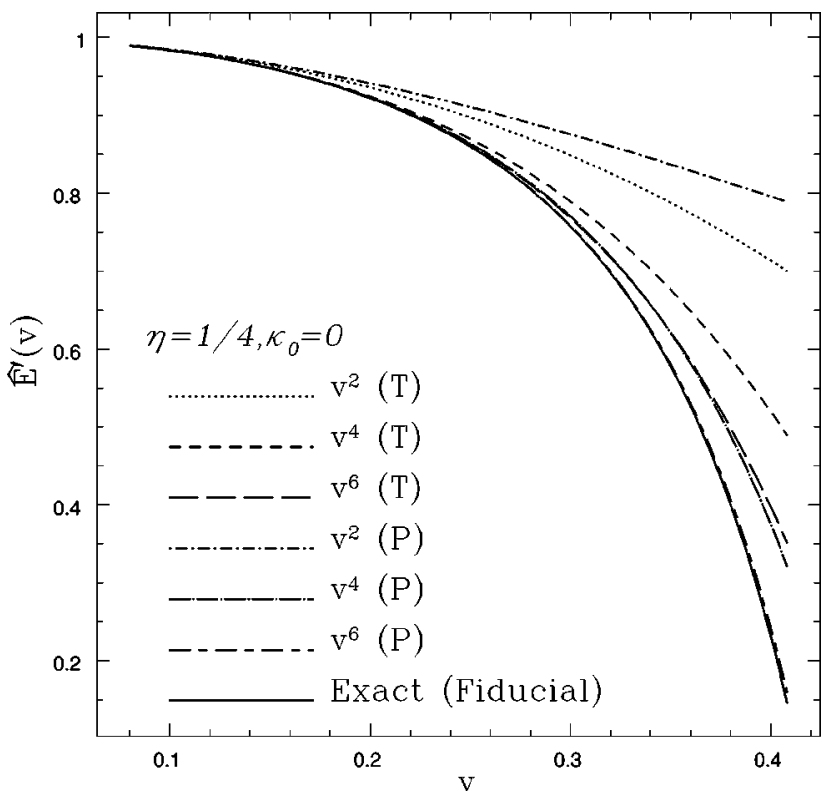

FIG. 5. Newton-normalized ( $v$-differentiated) energy functions in the comparable mass case. We compare the convergence of the $T$-approximants and $P$-approximants. Observe that the $P$-approximants converge much faster to the fiducial exact energy than the standard approximants.

The convergence tests performed in this section have shown at the visual level that the $P$-approximants behaved better than the $T$-ones. However, the real convergence criterion we are interested in is that defined by overlaps, to which we now turn.

\section{AMBIGUITY FUNCTION}

Central to our discussion is the ambiguity function which is a measure of the overlap of two wave forms that may differ from each other in not only their parameter values but

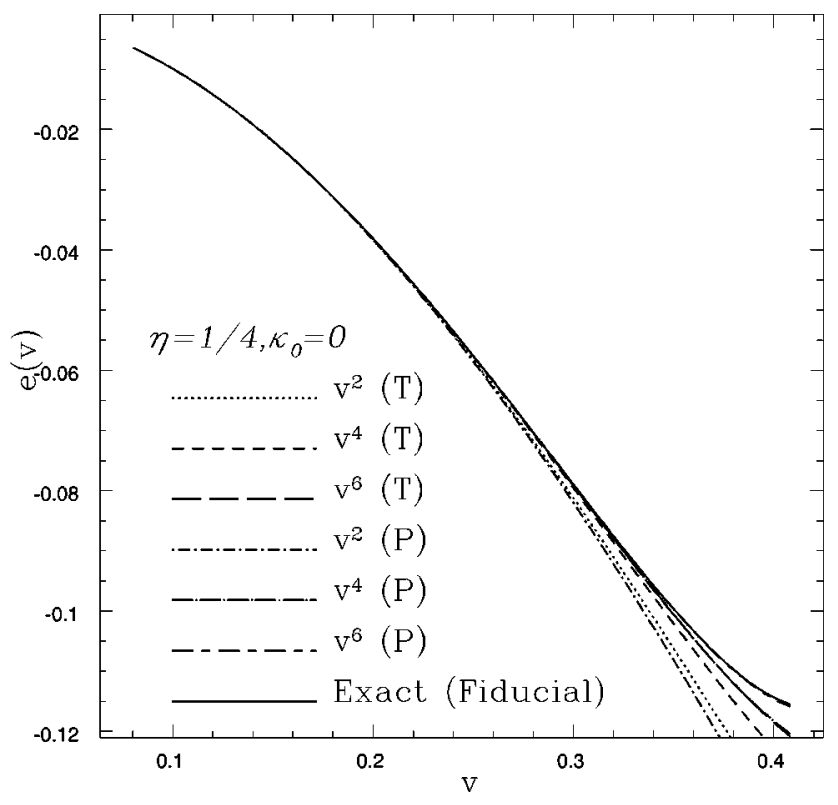

FIG. 6. Approximants to basic energy functions $e(v)$ are compared with the fiducial exact energy function. Convergence of $P$-approximants is apparent. also in their shape. For instance, one of them could be a first post-Newtonian signal corresponding to nonspinning stars parametrized by masses of the two stars and the other may be a second post-Newtonian inspiral wave form corresponding to spinning stars parametrized not only by the masses of the two stars but also by their spins. Let us therefore consider two wave forms $h\left(t ; \lambda_{k}, \phi_{h}\right)$ and $g\left(t ; \mu_{k}, \phi_{g}\right)$ where $\phi_{h}$ and $\phi_{g}$ denote the constant phases of the two wave forms, $\lambda_{k}$, $k=1, \ldots, n_{\lambda}$, and $\mu_{k}, k=1, \ldots, n_{\mu}$, are the dynamical parameters of the signals and $n_{\lambda}$ and $n_{\mu}$ are the corresponding number of parameters. The scalar product of these two wave forms is defined in Fourier space by

$$
\langle h, g\rangle\left(\tau ; \lambda_{k}, \mu_{k}\right) \equiv \int_{-\infty}^{\infty} \frac{d f e^{2 \pi i f \tau}}{S_{n}(f)} \widetilde{h}\left(f ; \lambda_{k}, \phi_{h}\right) \widetilde{g}^{*}\left(f ; \mu_{k}, \phi_{g}\right)
$$

where $\tau$ is the lag of one of the wave forms relative to the other; $\widetilde{h}\left(f ; \lambda_{k}, \phi_{h}\right)$ and $\widetilde{g}\left(f ; \mu_{k}, \phi_{g}\right)$ denote the Fourier transforms [37] of $h\left(t ; \lambda_{k}, \phi_{h}\right)$ and $g\left(t ; \mu_{k}, \phi_{g}\right)$, respectively, the asterisk denotes complex conjugation and $S_{n}(f)$ is the twosided noise power spectral density. The above scalar product is also the statistics of matched filtering (Wiener filter) which is the strategy used in detecting inspiraling binary signals. $S_{n}(f)$ being a (positive) real, even function of $f$ the scalar product (5.1) defines a real bilinear form in $h$ and $g$. We introduce also the norm $\|h\| \equiv \sqrt{\langle h, h\rangle}$. The ambiguity function $\mathcal{A}$ is defined as the value of the normalized scalar product maximized over the lag parameter $\tau$.

$$
\mathcal{A}\left(\lambda_{k}, \mu_{k}\right)=\max _{\tau, \phi} \frac{\langle h, g\rangle}{\|h\|\|g\|}
$$

where optimization over phases of the signal and the template is symbolically indicated by $\phi$ (see Appendix B for details). Here $\lambda_{k}$ can be thought of as the parameters of a signal while $\mu_{k}$ those of a template. The signal to noise ratio (SNR) for detecting a noise contaminated version of $h(t)$ with a Wiener filter built from $g(t-\tau)$ reads SNR $=\langle h, g\rangle /\|g\|$. Its maximum value is $\mathrm{SNR}_{\max }=\langle h, h\rangle /\|h\|=\|h\|$ when the time-translated $g$ is perfectly matched to the signal: $g(t-\tau)=h(t)$. Therefore $\mathcal{A}\left(\lambda_{k}, \mu_{k}\right)$ is the reduction in SNR obtained using a template that is not necessarily matched to the signal.

The dependences of $\mathcal{A}(\lambda, \mu)$ on both $\lambda$ and $\mu$ are important in designing detection strategies. The dependence on the signal parameters $\lambda$, given some template parameters $\mu$, allows one to define an optimal way of paving the template parameter space. The region in the signal parameter space for which a given template obtains SNRs larger than a certain value [38] (sometimes called the minimal match [39]) is the span of that template [40] and the templates should be so chosen that together they span the entire signal parameter space of interest with the least overlap of one other's spans. In our case, we are mainly interested in keeping the signal parameters $\lambda$ fixed, and varying the template ones $\mu$. In searching for a coalescing binary signal in the output of a detector one maximizes over a given bank of templates (i.e. over a dense lattice of $\mu$ values). Thus, the quantity of interest is the maximum of the ambiguity function over the entire 
parameter space of templates. This maximum, in the case of identical signals, occurs when the parameters of the template and the signal are equal and is equal to 1 . However, in reality the template wave forms are not identical to the fully general relativistic signal and hence the maximum overlap will in general be less than 1 (Schwarz inequality) and would occur not when the parameters are matched but when they are mismatched:

$$
\max _{\mu_{k}} \mathcal{A} \leqslant 1 .
$$

If the template wave forms are not "close" to signal wave forms, then it is reasonable to expect that the maximum occurs when $\left|\lambda_{k}-\mu_{k}\right|$ is fractionally rather large. In this case there is not only a substantial reduction in the maximum SNR that can be achieved by using such a bank of templates but there would also be a large systematic bias in the measurement of parameters. Using the terminology of the Introduction such template wave forms would be neither effectual nor faithful. For detection purposes we wish to construct effectual templates, i.e. templates having a large overlap after maximization over $\mu$. For parameter estimation we further need to construct faithful templates which have large overlaps when $\mu \simeq \lambda$. A practical (nonrigorous) criterion for faithfulness is that the "diagonal" ambiguity function $\mathcal{A}(\lambda, \lambda)$ be close to 1 .

Reduction in the overlap of template wave forms and true signals has an effect on the number of detectable events or, equivalently, loss in the detection probability of a signal of a given strength. For a given signal-to-noise ratio, the distance up to which a detector can detect depends primarily on the amplitude $h_{0}$ of the wave. Unavailability of a copy of the true signal means that the effective strength of the signal reduces from $h_{0}$ to $\mathcal{A} h_{0}$ and hence the span of a detector reduces by the factor $\mathcal{A}$. The number of events a detector can detect being proportional to the cube of the distance, a reduction in the overlap by a factor $\mathcal{A}$ means a drop in the number of detectable events, as compared to the case where a knowledge of the true wave form was available, by a factor $\mathcal{A}^{3}$. For instance, a $10 \%(20 \%)$ loss in the overlap would mean a $27 \%(50 \%)$ loss in the number of events [39]. The aim of PN calculations is to make this overlap as close to 1 as possible. If we demand that we should be able to detect with PN templates about $90 \%$ (99\%) of the signals that we would detect had we known the general relativistic signal, then we should have the overlap to be no less than about 0.965 (0.997).

As a model for noise above the seismic cutoff $f_{s}$ we use the expected noise power spectral density in the initial LIGO interferometer [41]:

$$
S_{n}(f)=\frac{S_{0}}{\alpha+3}\left[\alpha+2\left(\frac{f}{f_{0}}\right)^{2}+\left(\frac{f}{f_{0}}\right)^{-4}\right], \quad f>f_{s}
$$

where $S_{0}, \alpha$ and $f_{0}$ are constants that characterize the detector sensitivity, effective bandwidth and the frequency at which the detector noise is the lowest, respectively. In the case of initial LIGO $\alpha=2, f_{s}=40 \mathrm{~Hz}$ and $f_{0}=200 \mathrm{~Hz}$. Because of the fact that the noise is essentially infinite below the seismic cutoff $f_{s}$ and since we terminate the template
TABLE II. Overlap integrals of a test mass wave form whose Fourier transform is computed using stationary phase approximation with the same wave form but whose Fourier transform is computed using numerical fast Fourier transform. $n$ stands for the order of the Taylor approximant with $X$ denoting the exact wave form.

\begin{tabular}{lccc}
\hline \hline$n$ & $\mathrm{~A}_{0}$ & $\mathrm{~B}_{0}$ & $\mathrm{C}_{0}$ \\
\hline & $\left\langle T_{n}^{0}, T_{n}^{0}\right\rangle$ & $\left\langle T_{n}^{0}, T_{n}^{0}\right\rangle$ & $\left\langle T_{n}^{0}, T_{n}^{0}\right\rangle$ \\
\hline 0 & 1.000 & 0.995 & 0.967 \\
2 & 0.999 & 0.994 & 0.970 \\
4 & 0.999 & 0.990 & 0.955 \\
6 & 0.999 & 0.986 & 0.944 \\
8 & 0.999 & 0.988 & 0.945 \\
10 & 0.999 & 0.987 & 0.940 \\
$X$ & 0.999 & 0.978 & 0.918 \\
\hline \hline
\end{tabular}

wave forms when the velocity reaches that of the last stable orbit, the overlap integral (5.2) reduces to

$$
\begin{aligned}
& \mathcal{A}\left(\lambda_{k}, \mu_{k}\right) \\
& =\|h\|^{-1}\|g\|^{-1}
\end{aligned}
$$

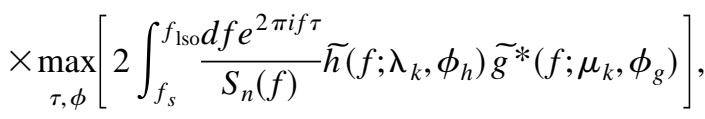

where $f_{\text {lso }}$ is the gravitational wave frequency corresponding to the last stable orbit. In order to compute the maximum overlap we proceed in the following manner. The evolution of phase as a function of time is obtained by inverting numerically $v$ in terms of $t$ from Eq. (2.14) and inserting the result in Eq. (2.15) and then Eq. (2.13). Though the iterative procedure in inverting $v$ in terms of $t$ is rather computationally intensive, we need to employ it since the inaccuracies introduced by the stationary phase approximation in computing the Fourier transform of the wave form increase with the order of approximation especially in the case of NS-BH and $\mathrm{BH}-\mathrm{BH}$ binaries. In Table II, we give a measure of the inaccuracies introduced by the stationary phase approximation at various post-Newtonian orders by computing the integral in Eq. (5.5) with $\widetilde{h}(f)$ being the fast Fourier transform and $\tilde{g}(f)$ being the stationary phase approximation of the same wave form. (The three cases $A_{0}, B_{0}$ and $C_{0}$ are defined below.) The worsened performance of the stationary phase approximation for massive systems is clearly linked to the fact that such systems emit fewer cycles in the effective bandwidth centered near $f_{0}$. Indeed, from our estimates above the gravitational wave signal from a $\left(10 M_{\odot}, 10 M_{\odot}\right)$ system shuts off at $f_{\text {lso }}^{\mathrm{GW}} \simeq 286 \mathrm{~Hz}$, which is not very much higher than $f_{0}$, and one should remember that the orbital evolution gets faster as one nears the lso so that fewer cycles are spent toward higher frequencies $f \leqslant f_{\text {lso }}^{\mathrm{GW}}$.

\section{RESULTS AND DISCUSSION}

Having in hand the ambiguity function to measure the closeness of two wave forms [42] we can use it to pursue at a quantitative level the analysis of the convergence of the 
sequence of approximants defined above.

Let us first consider the wave forms defined in the formal test mass limit where one keeps the $\eta$ factors in front of $E(v)$ and $F(v)$ but neglects the $\eta$ dependence in the Taylor coefficients of $\hat{E}^{\prime}(v)$ and $\hat{F}(v)$. Explicitly we mean the wave forms defined by eliminating (numerically) $v$ between

$$
\begin{gathered}
h^{A}(v)=C v^{2} \cos 2 \Phi_{A}(v), \\
t(v)=t_{c}-\frac{5}{32} \eta^{-1} m \int_{v}^{v_{\text {lso }}} d v v^{-9} \frac{\hat{E}_{A}^{\prime}(v ; \eta=0)}{\hat{F}_{A}(v ; \eta=0)} \\
\Phi_{A}(v)=\Phi_{c}-\frac{5}{32} \eta^{-1} \int_{v}^{v_{\text {lso }}} d v v^{-6} \frac{\hat{E}_{A}^{\prime}(v ; \eta=0)}{\hat{F}_{A}(v ; \eta=0)}
\end{gathered}
$$

in which $v_{\text {lso }}=v_{\text {lso }}(\eta=0)=1 / \sqrt{6}$. Note that the main purpose of the overlap computations made for this formal test mass limit is to compare quantitatively the convergence of the $P$-approximants to that of the $T$-ones. One should keep in mind that when studying below in the formal test mass limit $\left(1.4 m_{\odot}, 1.4 m_{\odot}\right)$ or $\left(10 m_{\odot}, 10 m_{\odot}\right)$ systems (for which $\eta$ takes its largest value) the absolute values of the overlaps are not reliable, though one assumes that the lessons learned from the $P / T$ comparison are. The absolute values of the overlaps for the $\left(1.4 m_{\odot}, 10 m_{\odot}\right)$ case are probably more reliable, but this is not clear as $\eta \simeq 0.1077$ is then only a factor 2.32 smaller than $\eta_{\max }=0.25$. This being said we wish to compare semi-maximized overlaps that we can denote for simplicity as

$$
\begin{aligned}
& \left\langle T_{n}^{0}\left(m_{1}, m_{2}\right), X^{0}\left(m_{1}, m_{2}\right)\right\rangle
\end{aligned}
$$

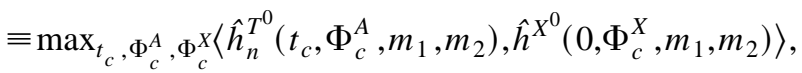

$$
\begin{aligned}
& \left\langle P_{n}^{0}\left(m_{1}, m_{2}\right), X^{0}\left(m_{1}, m_{2}\right)\right\rangle \\
& \quad \equiv \max _{t_{c}}, \Phi_{c}^{A}, \Phi_{c}^{X}\left\langle\hat{h}_{n}^{P^{0}}\left(t_{c}, \Phi_{c}^{A}, m_{1}, m_{2}\right), \hat{h}^{X^{0}}\left(0, \Phi_{c}^{X}, m_{1}, m_{2}\right)\right\rangle .
\end{aligned}
$$

Here the superscript 0 on $T, P$ or $X$ denotes the above defined formal $\eta=0$ limit of Taylor, Padé-type or exact wave forms, respectively [i.e. $A=T, P$ or $X$ in Eqs. (6.1)-(6.3)]. Here one considers only the same values for the two dynamical parameters of those signals [i.e. the explicit $m$ and $\eta$ appearing in Eqs. (6.1)-(6.3), here expressed in terms of $m_{1}$ and $m_{2}$ in order to psychologically minimize the formal inconsistency of setting $\eta=0$ in part of the formula and keeping it elsewhere] and maximize over the kinematical ones $t_{c}^{A}$, $\Phi_{c}^{A}, t_{c}^{X}, \Phi_{c}^{X}$. To maximize over the reference times, it is sufficient (as indicated above) to fix $t_{c}^{X}=0$ and maximize over $t_{c}^{A}=t_{c} \quad(=\tau$, the time lag). Maximizing over the reference phases is more subtle as the overlap depends separately on $\Phi_{c}^{A}$ and $\Phi_{c}^{X}$ and not only on their difference. There is, however, a computationally nonintensive way to do it which is based on a conceptually simple geometrical formulation of the problem (see Appendix B).
TABLE III. Faithfulness of the $T$ - and $P$-approximants in the test mass case. Values quoted are the minimax overlaps [cf. Eq. (B12)] together with the best possible overlaps [cf. Eq. (B11)] in parentheses.

\begin{tabular}{ccccccc}
\hline \hline$n$ & \multicolumn{2}{c}{$\mathrm{A}_{0}$} & \multicolumn{2}{c}{$\mathrm{B}_{0}$} & \multicolumn{2}{c}{$\mathrm{C}_{0}$} \\
\hline \multirow{4}{*}{4} & $\left\langle T_{n}^{0}, X^{0}\right\rangle$ & $\left\langle P_{n}^{0}, X^{0}\right\rangle$ & $\left\langle T_{n}^{0}, X^{0}\right\rangle$ & $\left\langle P_{n}^{0}, X^{0}\right\rangle$ & $\left\langle T_{n}^{0}, X^{0}\right\rangle$ & $\left\langle P_{n}^{0}, X^{0}\right\rangle$ \\
\hline \multirow{4}{*}{5} & 0.893 & 0.868 & 0.713 & 0.833 & 0.760 & 0.964 \\
& $(0.894)$ & $(0.868)$ & $(0.720)$ & $(0.838)$ & $(0.795)$ & $(0.984)$ \\
& 0.545 & 0.979 & 0.338 & 0.973 & 0.496 & 0.991 \\
6 & $(0.545)$ & $(0.980)$ & $(0.343)$ & $(0.974)$ & $(0.514)$ & $(0.998)$ \\
& 0.971 & 0.996 & 0.965 & 0.988 & 0.962 & 0.994 \\
7 & $(0.972)$ & $(0.996)$ & $(0.968)$ & $(0.990)$ & $(0.968)$ & $(0.999)$ \\
& 0.989 & 0.997 & 0.858 & 0.986 & 0.891 & 0.993 \\
8 & $(0.989)$ & $(0.998)$ & $(0.862)$ & $(0.988)$ & $(0.924)$ & $(0.999)$ \\
& 0.972 & 0.998 & 0.711 & 0.989 & 0.854 & 0.992 \\
9 & $(0.972)$ & $(0.999)$ & $(0.717)$ & $(0.992)$ & $(0.872)$ & $(0.999)$ \\
& 0.995 & 0.996 & 0.928 & 0.992 & 0.933 & 0.994 \\
& $(0.995)$ & $(0.996)$ & $(0.933)$ & $(0.994)$ & $(0.957)$ & $(0.999)$ \\
10 & 0.988 & - & 0.849 & - & 0.903 & - \\
& $(0.988)$ & $(-)$ & $(0.854)$ & $(-)$ & $(0.937)$ & $(-)$ \\
11 & 0.988 & 1.000 & 0.858 & 1.000 & 0.908 & 1.000 \\
& $(0.989)$ & $(1.000)$ & $(0.862)$ & $(1.000)$ & $(0.940)$ & $(1.000)$ \\
\hline \hline
\end{tabular}

Note that in Eqs. (6.4) the approximate template parameters are not optimized, but are taken to be equal to that of the exact signal. In other words we compare the faithfulness of the various approximants together with their convergence properties. The results are given in Table III, for $n=4-11$ [43] as well as for the Newtonian approximants for the purpose of comparison. The overlaps quoted are the minimax overlaps, Eq. (B12), together with the corresponding best overlaps, Eq. (B11), in parentheses below the minimax overlap. (The $P$-approximant $P_{4}^{3}$ corresponding to $n=7$ has a singularity in the region of interest and hence we have used the approximant $P_{3}^{4}$. The $P_{5}^{5}$-approximant too has a pole and we have not computed the overlaps in this case though if one desires one can compute other $P$-approximants, such as $P_{4}^{6}$ or $P_{6}^{4}$, at this order.) We consider three prototype cases, say case $A_{0}\left[\left(1.4 m_{\odot}, 1.4 m_{\odot}\right)\right]$, case $B_{0}\left[\left(1.4 m_{\odot}, 10 m_{\odot}\right)\right]$, and case $C_{0}\left[\left(10 m_{\odot}, 10 m_{\odot}\right)\right]$. We added an index zero to recall the fact that $\eta=0$ has been used in $\hat{E}^{\prime}$ and $\hat{F}$. (One should keep in mind the warning above that the numerical results for case $B_{0}$ are physically more reliable, while $A_{0}$ and $C_{0}$ are just mathematical ways of testing the convergence.)

We performed another convergence test (still in the formal $\eta \rightarrow 0$ limit) of a different nature. It is known in mathematics that one does not need to know in advance the limit of a sequence to test its convergence. One can instead use Cauchy's criterion which says (roughly) that the sequence converges if, given some distance function $d(h, g)$, $d\left(h_{n}, h_{m}\right) \rightarrow 0$, as both $n$ and $m$ get large. In our case we have a distance function [44] defined by the ambiguity function and we can compare the Cauchy convergence of the $T$ and $P$ approximants. Some results are given in Table IV where one exhibits the semi-maximized [in the sense of Eqs. (6.4)] best overlaps $\left\langle T_{n}^{0}, T_{n+1}^{0}\right\rangle$ versus $\left\langle P_{n}^{0}, P_{n+1}^{0}\right\rangle$, for $n$ $=4, \ldots, 11$, and the three prototype cases $A_{0}, B_{0}, C_{0}$. (As in 
TABLE IV. Cauchy convergence of the $T$ - and $P$-approximants in the test mass case. Values quoted are the best possible overlaps.

\begin{tabular}{lcccccc}
\hline \hline$n$ & \multicolumn{3}{c}{$\mathrm{A}_{0}$} & \multicolumn{2}{c}{$\mathrm{B}_{0}$} & \multicolumn{2}{c}{$\mathrm{C}_{0}$} \\
\hline & $\left\langle T_{n}^{0}, T_{n+1}^{0}\right\rangle$ & $\left\langle P_{n}^{0}, P_{n+1}^{0}\right\rangle$ & $\left\langle T_{n}^{0}, T_{n+1}^{0}\right\rangle$ & $\left\langle P_{n}^{0}, P_{n+1}^{0}\right\rangle$ & $\left\langle T_{n}^{0}, T_{n+1}^{0}\right\rangle$ & $\left\langle P_{n}^{0}, P_{n+1}^{0}\right\rangle$ \\
\hline 4 & 0.496 & 0.918 & 0.374 & 0.914 & 0.653 & 0.992 \\
5 & 0.528 & 0.984 & 0.330 & 0.986 & 0.541 & 0.999 \\
6 & 0.953 & 1.000 & 0.770 & 1.000 & 0.957 & 1.000 \\
7 & 0.988 & 0.999 & 0.913 & 0.997 & 0.982 & 0.998 \\
8 & 0.985 & 0.999 & 0.833 & 0.999 & 0.945 & 0.999 \\
9 & 0.997 & 0.999 & 0.965 & 0.994 & 0.985 & 0.999 \\
10 & 1.000 & - & 0.997 & - & 0.999 & - \\
\hline \hline
\end{tabular}

Table III where appropriate we have used the $P_{3}^{4}$-approximant instead of $P_{4}^{3}$. Since the $P_{5}^{5}$ approximant has a pole in the region of interest, the entries corresponding to $n=10$ are blank and the entries corresponding to $n=9$ are the overlaps $\left\langle P_{9}^{0}, P_{11}^{0}\right\rangle$.)

The last two tables show very clearly that the $P$-approximants converge much better than the $T$-ones and that they provide a much more faithful representation of the signal. To measure the effectualness of our approximants (in the technical sense defined above) and study the biases they can introduce, we also performed numerical calculations in which we maximized over all parameters, say

$$
\begin{aligned}
& \left\langle\left\langle T_{n}^{0}, X^{0}\right\rangle\right\rangle\left(m_{1}, m_{2}\right) \equiv \max _{m_{1}^{A}, m_{2}^{A}\left\langle T_{n}^{0}\left(m_{1}^{A}, m_{2}^{A}\right), X^{0}\left(m_{1}, m_{2}\right)\right\rangle,} \\
& \left\langle\left\langle P_{n}^{0}, X^{0}\right\rangle\right\rangle\left(m_{1}, m_{2}\right) \equiv \max _{m_{1}^{A}, m_{2}^{A}\left\langle P_{n}^{0}\left(m_{1}^{A}, m_{2}^{A}\right), X^{0}\left(m_{1}, m_{2}\right)\right\rangle,},
\end{aligned}
$$

while keeping track of the parameter values $m_{1}^{A}, m_{2}^{A}$ which, given the signal parameters $m_{1}, m_{2}$, maximize the overlaps. The results are presented in Table $\mathrm{V}$ for the three prototype cases $A_{0}, B_{0}, C_{0}$ and for the most important values (for the near future) of the order of approximation: $n=4,5$ and 6 . In this case the overlaps are the minimax overlaps.

Our test mass results sum up the general behavior of the different approximants pretty well. First let us note that even at $O\left(v^{11}\right) T$-approximants do not achieve the requisite overlap of 0.965 except in the case of light binaries. This is consistent with the concern often expressed in the literature about the need for higher order post-Newtonian wave forms.
In our view the most worrying aspect of the $T$-approximant is not that it does not obtain a high overlap but that the behavior of the approximant is oscillatory in nature. For instance, the $O\left(v^{6}\right) T$-wave form achieves an overlap, with the exact wave form, of about 0.96 which reduces at $O\left(v^{8}\right)$ to as low as 0.71 for system $B_{0}$ and 0.85 for system $C_{0}$ (though for system $A_{0}$ it maintains a level of 0.965 ), increases at $O\left(v^{9}\right)$ to about 0.93 for these systems and again drops back at $O\left(v^{11}\right)$ to 0.85 and 0.90 for systems $B_{0}$ and $C_{0}$, respectively. One clearly notices that $P$-approximants do not show such an erratic behavior. Recall that, in the test mass case, we are comparing a known exact wave form with an approximate signal model and hence the above conclusions are free from any prejudice. Though the second post-Newtonian $P$-approximant wave form is not a faithful signal model, at $5 / 2$ post-Newtonian order the $P$-approximant is a faithful signal model.

Moreover, $P$-approximants show an excellent Cauchy convergence as evident from Table IV. Notice that the $T$-approximants have a poor Cauchy convergence for systems $B_{0}$ and $C_{0}$. This makes them ill suited as faithful templates. $T$-approximants are not always effectual signal models either. Sometimes they do obtain overlaps larger than $96.5 \%$ but at the cost of producing a very large bias in the estimation of total mass. This is to be contrasted with the $P$-approximants which are effectual at $O\left(v^{4}\right)$ at the level of $99.7 \%$ or better at the cost of very little bias $(\delta \mathrm{m} / \mathrm{m}$ always less than 3.5\% and less than $1 \%$ in most cases). We have also computed the biases in the estimation of the parameter $\eta$ and there too we see a similar trend.

TABLE V. Effectualness of the $T$ - and $P$-approximants in the test mass case. Values quoted are the minimax overlaps together with the percentage bias in the estimation of total mass $100\left(1-\mathrm{m}^{A} / \mathrm{m}\right)$ in parentheses.

\begin{tabular}{lcccccc}
\hline \hline$n$ & \multicolumn{2}{c}{$\mathrm{A}_{0}$} & \multicolumn{2}{c}{$\mathrm{B}_{0}$} & \multicolumn{2}{c}{$\mathrm{C}_{0}$} \\
\hline & $\left\langle\left\langle T_{n}^{0}, X^{0}\right\rangle\right\rangle$ & $\left\langle\left\langle P_{n}^{0}, X^{0}\right\rangle\right\rangle$ & $\left\langle\left\langle T_{n}^{0}, X^{0}\right\rangle\right\rangle$ & $\left\langle\left\langle P_{n}^{0}, X^{0}\right\rangle\right\rangle$ & $\left\langle\left\langle T_{n}^{0}, X^{0}\right\rangle\right\rangle$ & $\left\langle\left\langle P_{n}^{0}, X^{0}\right\rangle\right\rangle$ \\
\hline 4 & 0.993 & 0.973 & 0.971 & 0.999 & 0.899 & 0.998 \\
& $(0.036)$ & $(-0.079)$ & $(-18.408)$ & $(-3.361)$ & $(-2.658)$ & $(-0.649)$ \\
5 & 0.824 & 0.996 & 0.986 & 0.999 & 0.806 & 0.999 \\
& $(-0.382)$ & $(-0.029)$ & $(-104.846)$ & $(-1.428)$ & $(-15.040)$ & $(-0.314)$ \\
6 & 0.990 & 1.000 & 0.983 & 0.997 & 0.988 & 0.999 \\
& $(0.039)$ & $(-0.004)$ & $(-2.875)$ & $(-0.040)$ & $(-0.605)$ & $(0.000)$ \\
\hline \hline
\end{tabular}



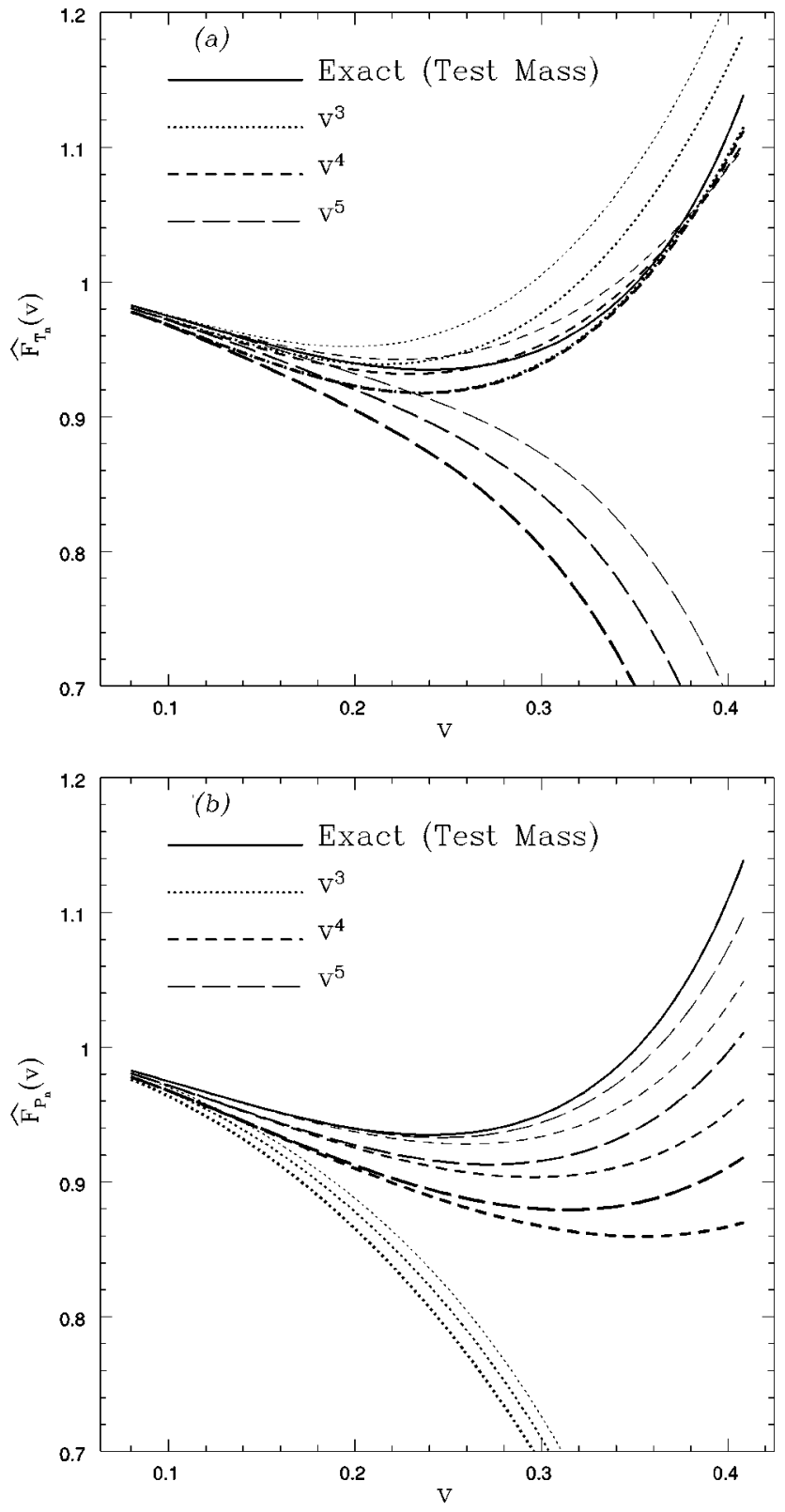

FIG. 7. Newton-normalized gravitational wave luminosity in the comparable mass case. Curves are plotted for three values of the mass ratio $(0,14 / 129.96$ and $1 / 4)$ with thicker curves corresponding to larger values of the mass ratio. (a) $T$-approximants and (b) $P$-approximants. For comparison we have also plotted the test particle flux. Note that the $T$-approximants as well as the $P$-approximants are continuous deformations of the test mass limit.

\section{ROBUSTNESS}

Up to this point in the paper we have mainly relied on the test mass limit to assess the quality of our approximants. In this section we shall try to go beyond this formal limit to check the robustness of our proposal under the turning on of $\eta$.

We can first use all the existing information about the comparable masses case and see whether turning on $\eta$ modifies in any way the trend we saw above. As a first test (a "visual" one) we plot in Fig. 7 the Newton-normalized flux functions $\hat{F}_{T_{n}}(v ; \eta), \hat{F}_{P_{n}}(v ; \eta)$ as a function of $v$, for the maximal value $\eta=\frac{1}{4}$ and for the cases where we know them, i.e. $n=2,3,4$ and 5. Using the same information we can also check the $\eta$ robustness of our Cauchy-convergence criterion. This is done in Table VI where we present the semimaximized best overlaps, Eq. (B11) $\left\langle P_{3}^{\eta}(m, \eta), P_{4}^{\eta}(m, \eta)\right\rangle$, $\left\langle P_{4}^{\eta}(m, \eta), P_{5}^{\eta}(m, \eta)\right\rangle \ldots,\left\langle P_{6}, P_{7}\right\rangle$ and compare them to their $T$ counterparts for the (real) cases $A, B$ and $C$.

We also made many attempts at testing the robustness of our conclusions when taking into account the existence of (unknown) higher-order $\eta$-dependent corrections. There is no really conclusive way of achieving such a task but here is our best attempt: Our starting point is to model an infinite number of (unknown) higher-order $P N$ corrections by just one (nonperturbative) parameter $\kappa_{0}$. As introduced in Eq. (4.11) above, $\kappa_{0}$ parametrizes our ignorance about the true location of the light ring [pole in $e(x)$ and $F(v)$ ]. Our 2PN Padé estimates gave us an $\eta$-corrected value $v_{\text {pole }}$, but we wish to consider here the possibility that maybe the true value is quite different from our estimate. More precisely Eq. (4.11) parametrizes the pole at $3 x_{\text {pole }}=\left(1-\kappa_{0} \eta\right)^{-1}$, while $3 x_{\text {pole }}^{P_{4}} \simeq 1.4312$ for $\eta=\frac{1}{4}$, corresponding to $\kappa_{0} \simeq+1.2051$. To explore a very large range of possibilities we shall consider that the true value of $\kappa_{0}$ (for $\left.\eta=0.25\right)$ might range between $\kappa_{0}=-1 \quad$ (meaning $3 x_{\text {pole }}=0.8$ ) and $\kappa_{0}=+2$ (meaning $3 x_{\text {pole }}=2.0$ ). In Table VII we compare the location of the last stable orbit $x_{1 \mathrm{so}} \equiv v_{\text {lso }}^{2}$ predicted by the $T$ and $P$-approximants to the energy function relative to the exact location $x_{\text {lso }}^{X}$. [For $t$-approximants the lso is defined by minimizing the standard energy function $E_{T_{n}}(v)$.] We see that $P$-approximants capture the location much better than the $T$-approximants.

Having chosen the range of $\kappa_{0}$ we shall consider, and adopting the definition (4.11) for the corresponding fiducial "exact" $e$ function, it remains to define a corresponding fiducial "exact" $f$ function, having the property that the cor-

TABLE VI. Cauchy convergence of the $T$ - and $P$-approximants in the comparable mass case. Values quoted are the best possible overlaps, Eq. (B11).

\begin{tabular}{ccccccc}
\hline \hline$n$ & \multicolumn{2}{c}{$\mathrm{A}$} & \multicolumn{2}{c}{$\mathrm{B}$} & \multicolumn{2}{c}{$\mathrm{C}$} \\
\hline & $\left\langle T_{n}^{\eta}, T_{n+1}^{\eta}\right\rangle$ & $\left\langle P_{n}^{\eta}, P_{n+1}^{\eta}\right\rangle$ & $\left\langle T_{n}^{\eta}, T_{n+1}^{\eta}\right\rangle$ & $\left\langle P_{n}^{\eta}, P_{n+1}^{\eta}\right\rangle$ & $\left\langle T_{n}^{\eta}, T_{n+1}^{\eta}\right\rangle$ & $\left\langle P_{n}^{\eta}, P_{n+1}^{\eta}\right\rangle$ \\
\hline 3 & 0.904 & 0.248 & 0.482 & 0.181 & 0.703 & 0.374 \\
4 & 0.411 & 0.863 & 0.338 & 0.872 & 0.545 & 0.985 \\
5 & 0.536 & 0.976 & 0.321 & 0.979 & 0.537 & 0.995 \\
6 & 0.946 & 0.982 & 0.736 & 0.996 & 0.928 & 0.999 \\
\hline \hline
\end{tabular}


TABLE VII. Location of the last stable circular orbit determined by the $T$ - and $P$-approximants in the finite mass case for different values of the parameter $\kappa_{0}$. At order $v^{2}$ the last stable orbit is not defined by $P$-approximants. At orders $v^{4}$ and beyond the $P$-approximants predict the location of lso pretty well.

\begin{tabular}{|c|c|c|c|c|}
\hline & \multicolumn{2}{|c|}{$\eta=1 / 4$} & \multicolumn{2}{|c|}{$\eta=14 /(11.4)^{2}$} \\
\hline \multirow[t]{3}{*}{$n$} & $x_{1 \mathrm{so}}^{T_{n}} / x_{1 \mathrm{so}}^{X}$ & $x_{1 \mathrm{so}}^{P_{n}} / x_{1 \mathrm{so}}^{X}$ & $x_{1 \mathrm{so}}^{T_{n}} / x_{\text {lso }}^{X}$ & $x_{1 \mathrm{so}}^{P_{n}} / x_{1 \mathrm{so}}^{X}$ \\
\hline & \multicolumn{4}{|c|}{$\kappa_{0}=-1$} \\
\hline & \multicolumn{2}{|c|}{$x_{1 \mathrm{so}}^{X}=0.1600$} & \multicolumn{2}{|c|}{$x_{1 \mathrm{so}}^{X}=0.1636$} \\
\hline 2 & 2.8852 & - & 2.9495 & - \\
\hline 4 & 1.5991 & 1.2415 & 1.5060 & 1.0976 \\
\hline \multirow[t]{3}{*}{6} & 1.2652 & 1.0205 & 1.2078 & 1.0035 \\
\hline & \multicolumn{4}{|c|}{$\kappa_{0}=47 / 39$} \\
\hline & \multicolumn{2}{|c|}{$x_{1 \mathrm{so}}^{X}=0.1986$} & \multicolumn{2}{|c|}{$x_{1 \mathrm{so}}^{X}=0.1792$} \\
\hline 2 & 2.3240 & - & 2.6934 & - \\
\hline 4 & 1.2881 & 1.0000 & 1.3752 & 1.0023 \\
\hline \multirow[t]{3}{*}{6} & 1.1020 & 1.0000 & 1.1408 & 1.0000 \\
\hline & \multicolumn{4}{|c|}{$\kappa_{0}=2$} \\
\hline & \multicolumn{2}{|c|}{$x_{1 \mathrm{so}}^{X}=0.2145$} & \multicolumn{2}{|c|}{$x_{1 \mathrm{so}}^{X}=0.1852$} \\
\hline 2 & 2.1517 & - & 2.6060 & - \\
\hline 4 & 1.1925 & 0.9258 & 1.3306 & 0.9698 \\
\hline 6 & 1.0568 & 1.0042 & 1.1186 & 1.0005 \\
\hline
\end{tabular}

responding $F$ function coincides, up to $O\left(v^{6}\right)$ terms, with the known $T_{5}$ expansion of $F$. To this effect the simplest proposal is to define first the $T_{11}$ (Taylor to $v^{11}$ ) expansion of $f_{X}^{\kappa_{0}}(v)$ by

$$
\begin{aligned}
T_{11}\left[f_{X}^{\kappa_{0}}\right] \equiv & T_{11}\left\{[ 1 - \sqrt { 3 ( 1 - \kappa _ { 0 } \eta ) } v ] \left[\sum_{k=0}^{5} A_{k}(\eta) v^{k}\right.\right. \\
& \left.\left.+\sum_{k=6}^{11}\left[A_{k}(0) v^{k}+B_{k}(0) v^{k} \ln v\right]\right]\right\},
\end{aligned}
$$

where $A_{k}(\eta), k \leqslant 5$, are given by Eq. (4.3), and the others $(\eta=0)$ by Eq. (4.2). Then we define the corresponding fiducial "exact" $f$ function by

$$
f_{X}^{\kappa_{0}}(v ; \eta) \equiv f_{P_{11}}(v ; \eta) \equiv \text { the RHS of Eq. (4.6). }
$$

Having defined some fiducial "exact" $e$ and $f$ functions we have correspondingly defined some "exact'" wave form $h_{X}^{\kappa_{0}}$ and, using the definitions above, both $T$-type and $P$-type approximants of this wave form. We are interested in knowing whether the $P$-approximants behave better than the $T$-ones even in presence of higher-order effects significantly different from the behavior expected from the 2PN Padé results. The results of this exercise are presented in Table VIII where one has computed the semi-optimized minimax overlaps $\left\langle P_{n}^{\eta}(m, \eta), X_{\kappa_{0}}^{\eta}(m, \eta)\right\rangle$ and $\left\langle T_{n}^{\eta}(m, \eta), X_{\kappa_{0}}^{\eta}(m, \eta)\right\rangle$ for the cases $A, B, C$, for $\kappa_{0}=-1,1.2051$ and 2 and for $n$ $=4,5,6$ and 7 . In order to test the effectiveness of the approximants, in Table IX we quote the fully optimized but minimax overlaps $\left\langle\left\langle P_{n}^{\eta}(m, \eta), X_{\kappa_{0}}^{\eta}(m, \eta)\right\rangle\right\rangle$ and $\left\langle\left\langle T_{n}^{\eta}(m, \eta), X_{\kappa_{0}}^{\eta}(m, \eta)\right\rangle\right\rangle$ again for the cases $A, B, C$, for $\kappa_{0}=-1,1.2051$ and 2 and for $n=4,5,6$ and 7 .

From Table VIII we clearly see that $T$-approximants fail to be faithful signal models even at the third post-Newtonian order. The second post-Newtonian wave form of this family would clearly fail to capture even $20 \%$ of all potential NS-NS events that would be detectable with the aid of a family of templates constructed out of $P$-approximants. Even when parameter values are extreme $\left(\kappa_{0}=-1\right.$, and very high masses) the presently available $5 / 2$ post-Newtonian energy and flux functions are sufficient to construct a faithful $P$-approximant.

We observe that except when the parameter values are extreme (very low value of $\kappa_{0}$ and high masses) $O\left(v^{5}\right)$ $P$-approximants are indeed good effectual signal models. In fact in all cases, but one, they obtain an overlap in excess of $99 \%$. Bias in the estimation of the total mass is at worst $7.6 \%$ and in many cases it is below $2 \%$. On the contrary standard second post-Newtonian approximants are not effectual in many cases; when they are effectual they often produce a relatively large bias. For instance, for system $B$, when $\kappa_{0}$ $=47 / 39$, the second post-Newtonian $T$-approximant acquires an overlap of 0.98 compared to 1.00 acquired by the $P$-approximant of the same order. However, the bias is $97 \%$ in the former case as compared to a tiny $1.1 \%$ in the latter case. Similarly, for $\kappa_{0}=2$, the 2.5 post-Newtonian $T$-approximant achieves an overlap of 0.988 at a bias of $75 \%$ while the $P$-approximant wave form achieves 0.996 overlap with practically no bias at all. The biases in the estimation of the $\eta$ parameter (not shown) are also pretty small when $P$-approximants are used as compared to $T$-approximants.

A word of caution is in order for those who desire to use standard post-Newtonian templates: A careful examination of the above tables reveals that the 2.5 post-Newtonian $T$-approximant systematically obtains poorer overlaps and larger biases. This is of course related to the fact that the $5 / 2$ post-Newtonian flux is very badly behaved (cf. Fig. 3). Hence one must never employ the 2.5 post-Newtonian $T$-approximant for searches. However, $P$-approximants do not suffer from this predicament. Indeed at $O\left(v^{5}\right)$ the $P$-wave form is an excellent effectual signal model. For all systems and parameters this model obtains an overlap of better than $99.5 \%$ at a bias less than $1.5 \%$.

\section{CONCLUSIONS}

In this work we have studied the convergence properties of various post-Newtonian templates to detect gravitational waves emitted by inspiraling compact binaries consisting of neutron stars and/or black holes. We have shown that the standard post-Newtonian filters, referred to as the $T$-approximants that are based on Taylor series, considered in the literature define a badly convergent sequence of approximants. Even at order $v^{11}$ the $T$-approximants only provide overlaps $\sim 0.86$ with the exact signal in the case of binaries consisting of (1.4-10) $M_{\odot}$ systems. Worse, the convergence of the sequence of $T$-approximants is oscillatory rather than monotonous. Our results on $T$-approximants con- 
TABLE VIII. Robustness of the $T$ - and $P$-approximants in the comparable mass case: faithfulness. Values quoted are the minimax overlaps together with the best possible overlaps, Eq. (B11), in parentheses. System D corresponds to a binary consisting of stars of masses $20 M_{\odot}$ and $1.4 M_{\odot}$. In this extreme mass ratio case the $P$-approximants at $O\left(v^{5}\right)$ are not faithful (overlaps $<96.5 \%$ ).

\begin{tabular}{|c|c|c|c|c|c|c|c|c|}
\hline \multirow[t]{2}{*}{$n$} & \multicolumn{2}{|c|}{ A } & \multicolumn{2}{|c|}{ B } & \multicolumn{2}{|c|}{$\mathrm{C}$} & \multicolumn{2}{|c|}{$\mathrm{D}$} \\
\hline & $\left\langle T_{n}^{\eta}, X^{\eta}\right\rangle$ & $\left\langle P_{n}^{\eta}, X^{\eta}\right\rangle$ & $\left\langle T_{n}^{\eta}, X^{\eta}\right\rangle$ & $\left\langle P_{n}^{\eta}, X^{\eta}\right\rangle$ & $\left\langle T_{n}^{\eta}, X^{\eta}\right\rangle$ & $\left\langle P_{n}^{\eta}, X^{\eta}\right\rangle$ & $\left\langle T_{n}^{\eta}, X^{\eta}\right\rangle$ & $\left\langle P_{n}^{\eta}, X^{\eta}\right\rangle$ \\
\hline & \multicolumn{8}{|c|}{$\kappa_{0}=-1$} \\
\hline 4 & $\begin{array}{c}0.601 \\
(0.602)\end{array}$ & $\begin{array}{c}0.875 \\
(0.875)\end{array}$ & $\begin{array}{c}0.882 \\
(0.887)\end{array}$ & $\begin{array}{c}0.735 \\
(0.742)\end{array}$ & $\begin{array}{c}0.826 \\
(0.860)\end{array}$ & $\begin{array}{c}0.825 \\
(0.841)\end{array}$ & $\begin{array}{c}0.467 \\
(0.476)\end{array}$ & $\begin{array}{c}0.696 \\
(0.705)\end{array}$ \\
\hline 5 & $\begin{array}{c}0.528 \\
(0.528)\end{array}$ & $\begin{array}{c}0.954 \\
(0.954)\end{array}$ & $\begin{array}{c}0.323 \\
(0.328)\end{array}$ & $\begin{array}{c}0.875 \\
(0.881)\end{array}$ & $\begin{array}{c}0.416 \\
(0.432)\end{array}$ & $\begin{array}{c}0.842 \\
(0.873)\end{array}$ & $\begin{array}{c}0.272 \\
(0.279)\end{array}$ & $\begin{array}{c}0.824 \\
(0.841)\end{array}$ \\
\hline \multirow[t]{2}{*}{6} & $\begin{array}{c}0.989 \\
(0.990)\end{array}$ & $\begin{array}{c}0.987 \\
(0.988)\end{array}$ & $\begin{array}{c}0.968 \\
(0.972)\end{array}$ & $\begin{array}{c}0.990 \\
(0.992)\end{array}$ & $\begin{array}{c}0.910 \\
(0.917)\end{array}$ & $\begin{array}{c}0.991 \\
(0.996)\end{array}$ & $\begin{array}{c}0.896 \\
(0.900)\end{array}$ & $\begin{array}{c}0.998 \\
(0.999)\end{array}$ \\
\hline & \multicolumn{8}{|c|}{$\kappa_{0}=47 / 39$} \\
\hline 4 & $\begin{array}{c}0.566 \\
(0.567)\end{array}$ & $\begin{array}{c}0.810 \\
(0.810)\end{array}$ & $\begin{array}{c}0.908 \\
(0.912)\end{array}$ & $\begin{array}{c}0.793 \\
(0.795)\end{array}$ & $\begin{array}{c}0.921 \\
(0.946)\end{array}$ & $\begin{array}{c}0.954 \\
(0.976)\end{array}$ & $\begin{array}{c}0.539 \\
(0.550)\end{array}$ & $\begin{array}{c}0.800 \\
(0.813)\end{array}$ \\
\hline 5 & $\begin{array}{c}0.553 \\
(0.554)\end{array}$ & $\begin{array}{c}0.983 \\
(0.983)\end{array}$ & $\begin{array}{c}0.335 \\
(0.339)\end{array}$ & $\begin{array}{c}0.975 \\
(0.975)\end{array}$ & $\begin{array}{c}0.490 \\
(0.509)\end{array}$ & $\begin{array}{c}0.989 \\
(0.999)\end{array}$ & $\begin{array}{c}0.283 \\
(0.290)\end{array}$ & $\begin{array}{c}0.961 \\
(0.965)\end{array}$ \\
\hline 6 & $\begin{array}{c}0.968 \\
(0.968)\end{array}$ & $\begin{array}{c}0.998 \\
(0.998)\end{array}$ & $\begin{array}{c}0.923 \\
(0.926)\end{array}$ & $\begin{array}{c}0.997 \\
(0.999)\end{array}$ & $\begin{array}{c}0.974 \\
(0.983)\end{array}$ & $\begin{array}{c}0.997 \\
(0.999)\end{array}$ & $\begin{array}{c}0.940 \\
(0.942)\end{array}$ & $\begin{array}{c}0.990 \\
(0.996)\end{array}$ \\
\hline & \multicolumn{8}{|c|}{$\kappa_{0}=2$} \\
\hline 4 & $\begin{array}{c}0.557 \\
(0.557)\end{array}$ & $\begin{array}{c}0.779 \\
(0.780)\end{array}$ & $\begin{array}{c}0.894 \\
(0.896)\end{array}$ & $\begin{array}{c}0.813 \\
(0.817)\end{array}$ & $\begin{array}{c}0.896 \\
(0.905)\end{array}$ & $\begin{array}{c}0.985 \\
(0.989)\end{array}$ & $\begin{array}{c}0.586 \\
(0.598)\end{array}$ & $\begin{array}{c}0.843 \\
(0.848)\end{array}$ \\
\hline 5 & $\begin{array}{c}0.563 \\
(0.563)\end{array}$ & $\begin{array}{c}0.989 \\
(0.989)\end{array}$ & $\begin{array}{c}0.339 \\
(0.344)\end{array}$ & $\begin{array}{c}0.995 \\
(0.995)\end{array}$ & $\begin{array}{c}0.507 \\
(0.531)\end{array}$ & $\begin{array}{c}0.978 \\
(0.996)\end{array}$ & $\begin{array}{c}0.286 \\
(0.294)\end{array}$ & $\begin{array}{c}0.992 \\
(0.992)\end{array}$ \\
\hline 6 & $\begin{array}{c}0.964 \\
(0.965)\end{array}$ & $\begin{array}{c}0.999 \\
(0.999)\end{array}$ & $\begin{array}{c}0.883 \\
(0.889)\end{array}$ & $\begin{array}{c}0.978 \\
(0.978)\end{array}$ & $\begin{array}{c}0.955 \\
(0.970)\end{array}$ & $\begin{array}{c}0.997 \\
(0.998)\end{array}$ & $\begin{array}{c}0.939 \\
(0.949)\end{array}$ & $\begin{array}{c}0.984 \\
(0.992)\end{array}$ \\
\hline
\end{tabular}

firm previous, less convincing arguments in the literature, which were either based on rough quantitative estimates or on numerical calculations based on the stationary phase approximation for Fourier transforms - an approximation that we have shown not to be sufficiently accurate for this purpose (see Table II).

We have defined a new sequence of approximants, referred to as the $P$-approximants, based on two ingredients: (i) the introduction, on theoretical ground, of two new energy-type and flux-type functions $e(v)$ and $f(v)$, instead of the conventionally used $E(v)$ and $F(v)$ and (ii) the systematic use of the Padé approximation for constructing successive approximants of $e(v)$ and $f(v)$. The new sequence of $P$-approximants has been shown to exhibit a systematically better convergence behavior than the $T$-approximants. The overlaps they achieve at a fixed post-Newtonian order are usually much higher, and the convergence is essentially monotonous instead of oscillatory (as pictorially described in Fig. 1 and mathematically measured by the overlaps quoted in Tables III, V, VIII, and IX). From our extensive study of the formal ' "test-mass limit'" $\eta \equiv m_{1} m_{2} /\left(m_{1}+m_{2}\right)^{2} \Rightarrow 0$, i.e. keeping overall $\eta$ factors but neglecting $\eta$ in the coefficients of the post-Newtonian expansions, it appears that the presently known $O(v / c)^{5}$-accurate post-Newtonian results allow one to construct approximants having overlaps larger than $96.5 \%$ (overlaps corresponding to $\kappa_{0}=47 / 39,2$ in Table IX and all, but one, overlaps in Table IX) with the exact signals.
Such overlaps are enough to guarantee that no more than $10 \%$ of signals may remain undetected. By contrast $(v / c)^{5}$-accurate $T$-approximants only give overlaps of $50 \%$, and sometimes even as low as $30 \%$, corresponding to a loss of $87.5 \%$ and $97 \%$ events, respectively. Our results are summarized in Fig. 8 where we have plotted the fraction of events which the templates constructed out of $T$ and $P$-approximants would detect relative to the total number of events that would have been detectable if we have had access to the true signal. We clearly notice the superiority of the $P$-approximants. Moreover, our computations indicate that the new templates entail only acceptably small biases in the estimation of signal parameters (see Tables V and IX). In the terminology introduced in the text, $P$-approximants are both more effectual (higher fully maximized overlaps) and more faithful (smaller biases) than the usual $T$-approximants. The above conclusions are primarily based on the study of the formal test-mass limit and assumes that turning on $\eta$ brings only a smooth deformation of what happens at $\eta \rightarrow 0$. We have also studied the effect of turning on $\eta(\eta \neq 0)$ in the coefficients of the post-Newtonian expansions. From all our checks it seems that the $\eta$ dependence is indeed smooth and should not alter the fact that the $P$-approximants have a better convergence than the $T$-ones. Our construction predicts that the last stable circular orbit is closer (i.e. larger orbital frequency) when $\eta \neq 0$ [see Eq. (3.23)]. This is good news because it improves the efficiency of $P$-approximants to be 
TABLE IX. Robustness of the $T$ - and $P$-approximants in the comparable mass case: effectualness. Values quoted are the minimax overlaps, Eq. (B12), together with the percentage bias in the estimation of total mass $100\left(1-m^{A} / m\right)$ in parentheses.

\begin{tabular}{|c|c|c|c|c|c|c|}
\hline \multirow[t]{2}{*}{$n$} & \multicolumn{2}{|c|}{ A } & \multicolumn{2}{|c|}{ B } & \multicolumn{2}{|c|}{$\mathrm{C}$} \\
\hline & $\left\langle\left\langle T_{n}^{\eta}, X^{\eta}\right\rangle\right\rangle$ & $\left\langle\left\langle P_{n}^{\eta}, X^{\eta}\right\rangle\right\rangle$ & $\left\langle\left\langle T_{n}^{\eta}, X^{\eta}\right\rangle\right\rangle$ & $\left\langle\left\langle P_{n}^{\eta}, X^{\eta}\right\rangle\right\rangle$ & $\left\langle\left\langle T_{n}^{\eta}, X^{\eta}\right\rangle\right\rangle$ & $\left\langle\left\langle P_{n}^{\eta}, X^{\eta}\right\rangle\right\rangle$ \\
\hline & \multicolumn{6}{|c|}{$\kappa_{0}=-1$} \\
\hline 4 & $\begin{array}{c}0.891 \\
(0.268)\end{array}$ & $\begin{array}{c}0.970 \\
(-0.089)\end{array}$ & $\begin{array}{c}0.962 \\
(-12.550)\end{array}$ & $\begin{array}{c}0.997 \\
(-7.582)\end{array}$ & $\begin{array}{c}0.881 \\
(-2.217)\end{array}$ & $\begin{array}{c}0.940 \\
(-2.779)\end{array}$ \\
\hline 5 & $\begin{array}{c}0.811 \\
(-0.400)\end{array}$ & $\begin{array}{c}0.989 \\
(-0.039)\end{array}$ & $\begin{array}{c}0.713 \\
(-26.491)\end{array}$ & $\begin{array}{c}0.996 \\
(-4.907)\end{array}$ & $\begin{array}{c}0.664 \\
(-15.860)\end{array}$ & $\begin{array}{c}0.932 \\
(-2.660)\end{array}$ \\
\hline \multirow[t]{2}{*}{6} & $\begin{array}{c}0.995 \\
(0.011)\end{array}$ & $\begin{array}{c}0.991 \\
(0.025)\end{array}$ & $\begin{array}{c}0.982 \\
(-0.869)\end{array}$ & $\begin{array}{c}0.999 \\
(-0.104)\end{array}$ & $\begin{array}{c}0.955 \\
(-0.999)\end{array}$ & $\begin{array}{c}0.998 \\
(0.080)\end{array}$ \\
\hline & \multicolumn{6}{|c|}{$\kappa_{0}=47 / 39$} \\
\hline 4 & $\begin{array}{c}0.868 \\
(0.214)\end{array}$ & $\begin{array}{c}0.965 \\
(-0.111)\end{array}$ & $\begin{array}{c}0.975 \\
(-6.961)\end{array}$ & $\begin{array}{c}0.999 \\
(-3.618)\end{array}$ & $\begin{array}{c}0.972 \\
(1.406)\end{array}$ & $\begin{array}{c}0.997 \\
(-1.524)\end{array}$ \\
\hline 5 & $\begin{array}{c}0.831 \\
(-0.429)\end{array}$ & $\begin{array}{c}0.999 \\
(-0.032)\end{array}$ & $\begin{array}{c}0.981 \\
(-97.241)\end{array}$ & $\begin{array}{c}1.000 \\
(-1.118)\end{array}$ & $\begin{array}{c}0.810 \\
(-23.654)\end{array}$ & $\begin{array}{c}0.999 \\
(-0.205)\end{array}$ \\
\hline 6 & $\begin{array}{c}0.984 \\
(0.025)\end{array}$ & $\begin{array}{c}0.998 \\
(0.000)\end{array}$ & $\begin{array}{c}0.989 \\
(1.004)\end{array}$ & $\begin{array}{c}0.999 \\
(-0.157)\end{array}$ & $\begin{array}{c}0.996 \\
(0.391)\end{array}$ & $\begin{array}{c}1.000 \\
(0.019)\end{array}$ \\
\hline & \multicolumn{6}{|c|}{$\kappa_{0}=2$} \\
\hline 4 & $\begin{array}{c}0.863 \\
(0.357)\end{array}$ & $\begin{array}{c}0.961 \\
(-0.121)\end{array}$ & $\begin{array}{c}0.968 \\
(-1.300)\end{array}$ & $\begin{array}{c}0.998 \\
(-2.874)\end{array}$ & $\begin{array}{c}0.996 \\
(1.699)\end{array}$ & $\begin{array}{c}0.997 \\
(-0.450)\end{array}$ \\
\hline 5 & $\begin{array}{c}0.836 \\
(-0.339)\end{array}$ & $\begin{array}{c}1.000 \\
(-0.025)\end{array}$ & $\begin{array}{c}0.983 \\
(-92.233)\end{array}$ & $\begin{array}{c}1.000 \\
(-0.056)\end{array}$ & $\begin{array}{c}0.988 \\
(-75.053)\end{array}$ & $\begin{array}{c}0.996 \\
(0.000)\end{array}$ \\
\hline 6 & $\begin{array}{c}0.981 \\
(0.032)\end{array}$ & $\begin{array}{c}0.999 \\
(0.000)\end{array}$ & $\begin{array}{c}0.993 \\
(0.688)\end{array}$ & $\begin{array}{c}0.995 \\
(-0.708)\end{array}$ & $\begin{array}{c}0.998 \\
(1.106)\end{array}$ & $\begin{array}{c}1.000 \\
(-0.154)\end{array}$ \\
\hline
\end{tabular}

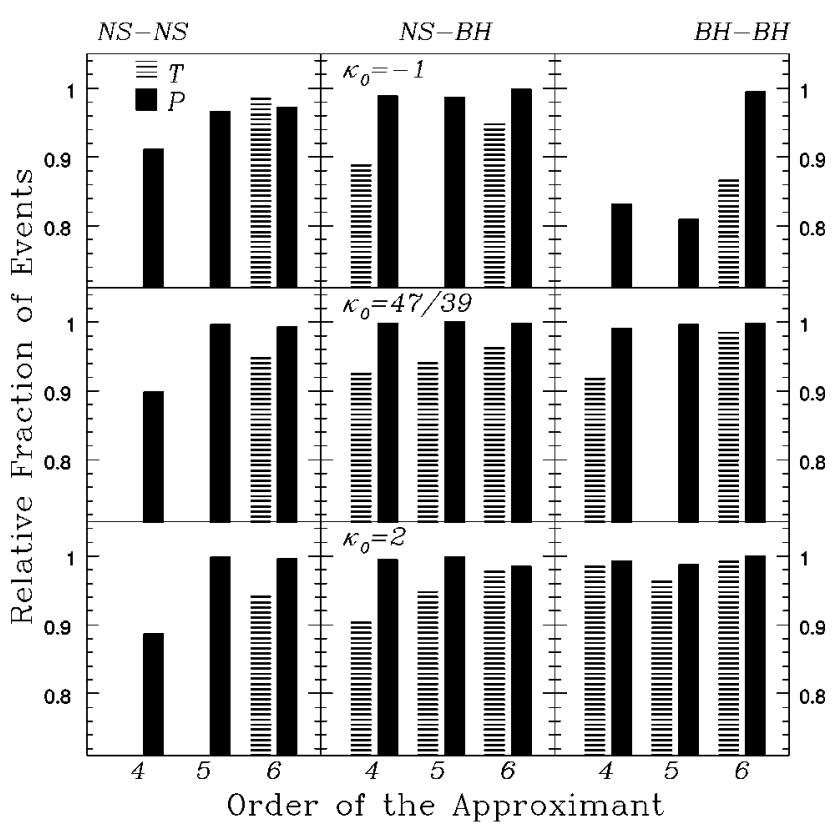

FIG. 8. Histogram of fraction of events accessible using $T$ - and $P$-approximants relative to the case when the true signal is known. used as filters for detectors having a fixed frequency band. However, we have no independent confirmation of this (favorable) dependence on $\eta$. We have tested the robustness of our conclusions against possible very drastic changes brought by (still unknown) $\eta$-dependent terms in the higher post-Newtonian coefficients. In the case where these extreme changes go in the opposite direction of what is suggested by presently known results (i.e. in the case $\kappa_{0}=-1$ ), we find that the overlaps are worsened compared to our best estimate range $\left(\kappa_{0}=47 / 39\right)$. This shows that it is important to extend the presently available $O\left(v^{5}\right)$ post-Newtonian results to the third post-Newtonian level (notably for the equations of motion) [25]. This will allow one to check whether the $\eta$ dependence of the 2.5 post-Newtonian results that we use is typical of the higher terms (as our method assumes) or exhibits some abnormal behavior for some unforeseeable reason. When third post-Newtonian results are available it is clearly still advisable to use the $P$-approximants: They have consistently higher overlaps and lower biases (cf. see Table IX).

In this study we have only considered the noise power spectral density corresponding to initial LIGO interferometers. Naturally, one must study other cases as well. Based on the current study we can be confident that in all cases the 
$P$-approximant wave forms will fare much better compared to the standard post-Newtonian ones. However, their performance in absolute terms needs to be reassessed since other interferometers, such as VIRGO, GEO600, and enhanced LIGO, happen to have effective bandwidths and the frequency of maximum sensitivity somewhat different from initial LIGO. In addition, one must also address the performance of $P$-approximate wave forms with regard to parameter estimation.

\section{ACKNOWLEDGMENTS}

It is a pleasure to thank Eric Poisson for providing the numerical test mass flux. B.R.I. thanks the Institut des Hautes Etudes Scientifiques, University of Wales Cardiff, and the Albert Einstein Institute, Potsdam, while B.S.S. thanks the Raman Research Institute and Institut des Hautes Etudes Scientifique for hospitality during different phases of this work. This work was supported in part by NSF grant PHY-9424337. B.S.S. thanks Kip Thorne and his group for useful conversations.

\section{APPENDIX A: PADÉ APPROXIMANTS}

A Padé approximant to the truncated Taylor series expansion of a function is a rational polynomial with the same number of coefficients as the latter. The coefficients of the Padé approximant are uniquely determined by reexpanding the Padé approximant to the same order as the truncated Taylor series and demanding that the two agree. In our study we use a continued fraction form of the (near diagonal) Padé approximant instead of the usual rational polynomial.

Let $S_{n}(v)=a_{0}+a_{1} v+\cdots+a_{n} v^{n}$ be a truncated Taylor series. A Padé approximant of the function whose Taylor approximant to order $v^{n}$ is $S_{n}$ is defined by two integers $m, k$ such that $m+k=n$. If $T_{n}[\cdots]$ denotes the operation of expanding a function in Taylor series and truncating it to accuracy $v^{n}$ (included), the $P_{k}^{m}$ Padé approximant of $S_{n}$ is defined by

$$
P_{k}^{m}(v)=\frac{N_{m}(v)}{D_{k}(v)}, \quad T_{n}\left[P_{m}^{k}(v)\right] \equiv S_{n}(v),
$$

where $N_{m}$ and $D_{k}$ are polynomials in $v$ of order $m$ and $k$, respectively. If one assumes that $D_{k}(v)$ is normalized so that
$D_{k}(0)=1$, i.e. $D_{k}(v)=1+q_{1} v+\cdots$, one shows that Padé approximants are uniquely defined by Eq. (A1). Note that, trivially, $P_{0}^{n}\left[S_{n}\right] \equiv S_{n}$ which indicates that Padé approximants are really useful when $k \neq 0$. Actually, it seems that in many cases the most useful Padé approximants are the ones near the "diagonal," $m=k$, i.e. $P_{m}^{m}$ if $n=2 m$ is even, and $P_{m}^{m+1}$ or $P_{m+1}^{m}$ if $n=2 m+1$ is odd. In this work we shall use, except when specified otherwise, the diagonal $\left(P_{m}^{m}\right)$ and the "subdiagonal" $\left(P_{m+1}^{m}\right)$ approximants. For instance, the $P_{4}^{3}$-approximant of the flux function has a pole and therefore we use instead the $P_{3}^{4}$-approximant. The diagonal $\left(P_{m}^{m}\right)$ or subdiagonal $\left(P_{m+1}^{m}\right)$ Padé approximants can be conveniently written in a continued fraction form (see e.g. [45]). For example, given

$$
S_{2}(v)=a_{0}+a_{1} v+a_{2} v^{2}
$$

one looks for

$$
P_{1}^{1}(v)=\frac{c_{0}}{1+\frac{c_{1} v}{1+c_{2} v}}=c_{0} \frac{1+c_{2} v}{1+\left(c_{1}+c_{2}\right) v}
$$

and given

$$
S_{3}(v)=a_{0}+a_{1} v+a_{2} v^{2}+a_{3} v^{3}
$$

one looks for

$$
P_{2}^{1}(v)=\frac{c_{0}}{1+\frac{c_{1} v}{1+\frac{c_{2} v}{1+c_{3} v}}}=c_{0} \frac{1+\left(c_{2}+c_{3}\right) v}{1+\left(c_{1}+c_{2}+c_{3}\right) v+c_{1} c_{3} v^{2}}
$$

The main advantage of using the continued fraction representations is that the lower order coefficients $c_{k}$ remain unchanged as we increase the order of the polynomial being approximated. This is not true for the coefficients of the polynomials $N_{m}, D_{k}$ in Eq. (A1). [This is easily seen by comparing Eqs. (A3) and (A5).] The $c_{k}$ 's are algorithmically obtainable in terms of the coefficients $a_{\ell}$ in $S_{n}$ with $\ell \leqslant k$. For instance,

$$
\begin{gathered}
c_{0}=a_{0}, \\
c_{1}=-\frac{a_{1}}{c_{0}}, \\
c_{2}=-\frac{c_{0} c_{1}^{2}-a_{2}}{c_{0} c_{1}}, \\
c_{3}=-\frac{c_{0} c_{1}\left(c_{2}+c_{1}\right)^{2}+a_{3}}{c_{0} c_{1} c_{2}}, \\
c_{4}=-\frac{c_{0} c_{1}\left(c_{2}+c_{1}\right)^{3}+c_{0} c_{1} c_{2} c_{3}\left(c_{3}+2 c_{2}+2 c_{1}\right)-a_{4}}{c_{0} c_{1} c_{2} c_{3}},
\end{gathered}
$$




$$
\begin{gathered}
c_{5}=-\frac{c_{0} c_{1}\left[\left(c_{2}+c_{1}\right)^{2}+c_{2} c_{3}\right]^{2}+c_{0} c_{1} c_{2} c_{3}\left(c_{4}+c_{3}+c_{2}+c_{1}\right)^{2}+a_{5}}{c_{0} c_{1} c_{2} c_{3} c_{4}}, \\
c_{6}=-\frac{1}{c_{0} c_{1} c_{2} c_{3} c_{4} c_{5}}\left\{c_{0} c_{1}\left(c_{1}+c_{2}\right)^{5}+c_{0} c_{1} c_{2} c_{3}\left[\left(c_{1}+c_{2}+c_{3}\right)^{3}+3\left(c_{1}+c_{2}\right)^{3}+3 c_{2} c_{3}\left(c_{1}+c_{2}\right)+c_{3}\left(c_{2}-c_{1}\right)\right]\right. \\
\left.+c_{0} c_{1} c_{2} c_{3} c_{4}\left[\left(c_{1}+c_{2}+c_{3}+c_{4}\right)^{2}+2\left(c_{1}+c_{2}+c_{3}\right)^{2}+c_{3}\left(c_{4}-2 c_{1}\right)\right]+c_{0} c_{1} c_{2} c_{3} c_{4} c_{5}\left[c_{5}+2\left(c_{4}+c_{3}+c_{2}+c_{1}\right)\right]-a_{6}\right\} .
\end{gathered}
$$

The expressions for coefficients $c_{7}, c_{8}, \ldots$ are long and not listed here. Explicitly in terms of the $a$ 's some of the above coefficients are

$$
\begin{gathered}
c_{0}=a_{0}, \\
c_{1}=-\frac{a_{1}}{a_{0}}, \\
c_{2}=-\frac{a_{2}}{a_{1}}+\frac{a_{1}}{a_{0}}, \\
c_{3}=\frac{a_{0}\left(a_{1} a_{3}-a_{2}^{2}\right)}{a_{1}\left(a_{1}^{2}-a_{2} a_{0}\right)} .
\end{gathered}
$$

A few other properties of the Padé approximants are useful to notice, such as

$$
\begin{gathered}
P_{k}^{m}\left[T_{n}[f]\right]=\left(P_{m}^{k}\left[T_{n}\left[f^{-1}\right]\right]\right)^{-1}, \\
P_{m}^{m+\delta}\left[S_{n}\right]=a_{0}+a_{1} v P_{m+\delta}^{m}\left[\widetilde{S}_{n-1}\right],
\end{gathered}
$$

where $\delta=0$ or 1 and where $\widetilde{S_{n-1}}$ is defined by "factoring after $v$ ": $S_{n}=a_{0}+a_{1} v \widetilde{S_{n-1}}$. Equation (A9) shows how to obtain the superdiagonal Padé approximant of $S_{n}$ from the continued fraction approximants $\left(\equiv P_{m+\delta}^{m}\right)$ of $\widetilde{S_{n-1}}$. This can be iterated to $P_{n}^{n+2}$, etc. [An alternative way would be, from Eq. (A8), to work with the inverse of the series $S_{n}$.]

\section{APPENDIX B: OPTIMIZING OVER THE PHASES}

The "exact" (label $X$ ) and approximate (label $A$ ) template wave forms have the form

$$
\begin{aligned}
& h^{X}\left(t ; t_{c}^{X}, \phi_{c}^{X}, C^{X}\right)=C^{X} a\left(t-t_{c}^{X}\right) \cos \left[\phi_{c}^{X}+\phi^{X}\left(t-t_{c}^{X}\right)\right], \\
& h^{A}\left(t ; t_{c}^{A}, \phi_{c}^{A}, C^{A}\right)=C^{A} a\left(t-t_{c}^{A}\right) \cos \left[\phi_{c}^{A}+\phi^{A}\left(t-t_{c}^{A}\right)\right],
\end{aligned}
$$

where we denoted $\phi_{c}^{X} \equiv 2 \Phi_{c}^{X}, \phi^{X}(t) \equiv 2 \Phi^{X}(t)$, etc. The normalized overlap between $h^{X}$ and $h^{A}$ depends on the time difference $t_{c}^{A}-t_{c}^{X}$ and (separately) on the two phases $\phi_{c}^{X}$, $\phi_{c}^{A}$. Here, we show how, for any given time lag $\tau=t_{c}^{A}-t_{c}^{X}$ (i.e. after having fixed, for instance, $t_{c}^{X}=0, t_{c}^{A}=\tau$ ), one can maximize the overlap over the two phases $\phi_{c}^{A}, \phi_{c}^{X}$.

To solve this maximization problem [46] it is useful to think in geometrical terms: Each wave form $h(t)$ is seen as a "vector" $h$ in an infinite-dimensional vector space $\mathcal{W}$ en- dowed with the Euclidean metric defined by the (Wiener) scalar product. For any given $t_{c}^{X}, t_{c}^{A}$, one sees by expanding the cosines by the usual addition formula that the twoparameter family of "vectors" $h^{A}\left(C^{A}, \phi_{c}^{A}\right)$ span a 2-plane, i.e. a two-dimensional linear subspace of $\mathcal{W}$. More explicitly, an unnormalized basis of this 2-plane is $h_{1}^{A}, h_{2}^{A}$ with

$$
h_{1}^{A} \equiv h^{A}\left(C^{A}=1, \phi_{c}^{A}=0\right), \quad h_{2}^{A} \equiv h^{A}\left(C^{A}=1, \phi_{c}^{A}=\frac{\pi}{2}\right),
$$

the generic vector in the 2-plane being $h^{A}\left(\lambda^{A}\right)=\lambda_{1}^{A} h_{1}^{A}$ $+\lambda_{2}^{A} h_{2}^{A}$ with $\lambda_{1}^{A}=C^{A} \cos \phi_{c}^{A}, \lambda_{2}^{A}=C^{A} \sin \phi_{c}^{A}$. Similarly, the two-parameter family of "exact" vectors can be written as $h^{X}\left(\lambda^{X}\right)=\lambda_{1}^{X} h_{1}^{X}+\lambda_{2}^{X} h_{2}^{X}$ with the same definitions as above with the label $A$ being changed into $X$. Optimizing over the phases means finding the maximum over the $\lambda^{A}$ and $\lambda^{X}$ of

$$
\cos \theta_{A X}=\left\langle\hat{h}^{A}, \hat{h}^{X}\right\rangle \equiv \frac{\left\langle\lambda_{1}^{A} h_{1}^{A}+\lambda_{2}^{A} h_{2}^{A}, \lambda_{1}^{X} h_{1}^{X}+\lambda_{2}^{X} h_{2}^{X}\right\rangle}{\left\|\lambda_{1}^{A} h_{1}^{A}+\lambda_{2}^{A} h_{2}^{A}\right\|\left\|\lambda_{1}^{X} h_{1}^{X}+\lambda_{2}^{X} h_{2}^{X}\right\|},
$$

where $\hat{h}^{A}$ denotes the unit vector $h^{A}(\lambda) /\left\|h^{A}(\lambda)\right\|$.

Directly attempting to maximize $\cos \theta_{A X}\left(\lambda^{A}, \lambda^{X}\right)$ is very cumbersome. The problem is, however, reduced to an easy one if one introduces orthonormalized bases in both 2planes: say $\left(e_{1}^{A}, e_{2}^{A}\right)$ in the $A$-(2-plane) and $\left(e_{1}^{X}, e_{2}^{X}\right)$ in the $X$-one, with $\left\langle e_{a}^{A}, e_{b}^{A}\right\rangle=\delta_{a b}=\left\langle e_{a}^{X}, e_{b}^{X}\right\rangle ; a, b=1,2$. For instance, these orthonormalized bases can be defined as

$$
\begin{gathered}
e_{1}^{A} \equiv\left\|h_{1}^{A}\right\|^{-1} h_{1}^{A}, \\
e_{2}^{A} \equiv\left\|h_{1}^{A}\right\|^{-1}\left[\left\|h_{1}^{A}\right\|^{2}\left\|h_{2}^{A}\right\|^{2}-\left\langle h_{1}^{A}, h_{2}^{A}\right\rangle^{2}\right]^{-1 / 2} \\
\times\left\{\left\|h_{1}^{A}\right\|^{2} h_{2}^{A}-\left\langle h_{1}^{A}, h_{2}^{A}\right\rangle h_{1}^{A}\right\},
\end{gathered}
$$

for the $A$-plane and similarly for the $X$-plane.

The overlap (B3) is then the scalar product between two unit vectors (one in each plane) which can be parametrized as $\quad \cos \theta_{\alpha \beta}=\left\langle e_{\alpha}^{A}, e_{\beta}^{X}\right\rangle \quad$ where $\quad e_{\alpha}^{A}=\cos \alpha e_{1}^{A}+\sin \alpha e_{2}^{A}$, $e_{\beta}^{X}=\cos \beta e_{1}^{X}+\sin \beta e_{2}^{X}$. Let $P_{X}$ denote the orthogonal projector onto the $X$-plane, and $p_{\alpha}$ denote the orthogonal projection of $e_{\alpha}^{A}$, i.e. explicitly

$$
p_{\alpha}=P_{X}\left(e_{\alpha}^{A}\right)=\left\langle e_{\alpha}^{A}, e_{1}^{X}\right\rangle e_{1}^{X}+\left\langle e_{\alpha}^{A}, e_{2}^{X}\right\rangle e_{2}^{X} .
$$

The scalar product $\left\langle e_{\alpha}^{A}, e_{\beta}^{X}\right\rangle$ is equal to $\left\langle p_{\alpha}, e_{\beta}^{X}\right\rangle$. It is maximized over $\beta$ when $e_{\beta}^{X}$ is parallel to $p_{\alpha}$, in which case its 
value is the norm of $p_{\alpha}$. This shows that the maximum of $\cos \theta_{A X}$ is equal to the maximum over $\alpha$ of the norm of $p_{\alpha}$ :

$$
\left(\cos \theta_{A X}\right)_{\max }=\max _{\alpha}\left\|p_{\alpha}\right\| .
$$

On the other hand,

$$
p_{\alpha}=\cos \alpha p_{1}+\sin \alpha p_{2},
$$

where

$$
\begin{aligned}
& p_{1} \equiv P_{X}\left(e_{1}^{A}\right)=\left\langle e_{1}^{A}, e_{1}^{X}\right\rangle e_{1}^{X}+\left\langle e_{1}^{A}, e_{2}^{X}\right\rangle e_{2}^{X}, \\
& p_{2} \equiv P_{X}\left(e_{2}^{A}\right)=\left\langle e_{2}^{A}, e_{1}^{X}\right\rangle e_{1}^{X}+\left\langle e_{2}^{A}, e_{2}^{X}\right\rangle e_{2}^{X} .
\end{aligned}
$$

In geometrical terms, $p_{\alpha}$ describes, as $\alpha$ varies, and $e l$ lipse in the $X$-plane (the projection of the circle $e_{\alpha}$ $=\cos \alpha e_{1}+\sin \alpha e_{2}$ ) and the maximum projection onto the $X$-plane corresponds to the semi-major axis. The square $\left\|p_{\alpha}\right\|^{2}=\left\langle p_{\alpha}, p_{\alpha}\right\rangle$ reads

$$
\left\|p_{\alpha}\right\|^{2}=A \cos ^{2} \alpha+B \sin ^{2} \alpha+2 C \cos \alpha \sin \alpha,
$$

where

$$
\begin{gathered}
A \equiv\left\|p_{1}\right\|^{2}=\left\langle e_{1}^{A}, e_{1}^{X}\right\rangle^{2}+\left\langle e_{1}^{A}, e_{2}^{X}\right\rangle^{2}, \\
B \equiv\left\|p_{2}\right\|^{2}=\left\langle e_{2}^{A}, e_{1}^{X}\right\rangle^{2}+\left\langle e_{2}^{A}, e_{2}^{X}\right\rangle^{2}, \\
C \equiv\left\langle p_{1}, p_{2}\right\rangle=\left\langle e_{1}^{A}, e_{1}^{X}\right\rangle\left\langle e_{2}^{A}, e_{1}^{X}\right\rangle+\left\langle e_{1}^{A}, e_{2}^{X}\right\rangle\left\langle e_{2}^{A}, e_{2}^{X}\right\rangle .
\end{gathered}
$$

Maximizing over $\alpha$ is now easy [using $\cos ^{2} \alpha=(1$ $+\cos 2 \alpha) / 2, \quad \sin ^{2} \alpha=(1-\cos 2 \alpha) / 2, \quad 2 \sin \alpha \cos \alpha=\sin 2 \alpha$ and maximizing over $2 \alpha$ ] and yields finally

$$
\left(\cos \theta_{A X}\right)_{\max }=\left\{\frac{A+B}{2}+\left[\left(\frac{A-B}{2}\right)^{2}+C^{2}\right]^{1 / 2}\right\}^{1 / 2} .
$$

Inserting the definitions of the orthonormalized vectors, Eq. (B4), into the definitions, Eq. (B10), of $A, B$ and $C$ can allow one to express $\left(\cos \theta_{A X}\right)_{\max }$ only in terms of various scalar products of the initial vectors $h_{1}^{A}, h_{2}^{A}, h_{1}^{X}, h_{2}^{X}$. It is easily checked that the final answer does not depend on the choice of basis in the $A$ - and $X$-planes, and can (if wished) be expressed only in terms of the "2-forms" $\omega^{A} \equiv h_{1}^{A} \wedge h_{2}^{A}$ and $\omega^{X} \equiv h_{1}^{X} \wedge h_{2}^{X}$ (and of the Euclidean structure of $\mathcal{W}$ ).

The result, Eq. (B11), gives the best possible overlap when optimizing separately over the phases of the exact and approximate signals. This gives the mathematical measure of the closeness of the two wave forms. However, in practice we do not have access to the phase of the exact signal. It might happen that the latter phase, i.e. equivalently the angle $\beta$, is not optimum. Therefore, a physically more relevant measure of the closeness of the two wave forms (especially for the purpose of detection) is obtained by first optimizing over $\alpha$ (the parameter we can dial) and then considering that $\beta$ has the worst possible value. In terms of the geometric reasoning given above one finds that the worst possible case corresponds to the semi-minor axis of the ellipse given by Eq. (B9), i.e.

$$
\min _{\beta} \max _{\alpha}\left(\cos \theta_{A X}\right)=\left\{\frac{A+B}{2}-\left[\left(\frac{A-B}{2}\right)^{2}+C^{2}\right]^{1 / 2}\right\}^{1 / 2} .
$$

In our simulations we considered both measures of the closeness of the two signals. We use Eq. (B11) when we study the mathematical convergence and we use Eq. (B12) when we are interested in the detection. We shall refer to Eq. (B11) as the best overlap and Eq. (B12) as the minimax overlap.
[1] E. Poisson, Phys. Rev. D 47, 1497 (1993).

[2] C. Cutler, L. S. Finn, E. Poisson, and G. J. Sussmann, Phys. Rev. D 47, 1511 (1993).

[3] H. Tagoshi and T. Nakamura, Phys. Rev. D 49, 4016 (1994).

[4] M. Sasaki, Prog. Theor. Phys. 92, 17 (1994).

[5] H. Tagoshi and M. Sasaki, Prog. Theor. Phys. 92, 745 (1994).

[6] L. Blanchet, T. Damour, B. R. Iyer, C. M. Will, and A. G. Wiseman, Phys. Rev. Lett. 74, 3515 (1995).

[7] L. Blanchet, T. Damour, and B. R. Iyer, Phys. Rev. D 51, 5360 (1995).

[8] C. M. Will and A. G. Wiseman, Phys. Rev. D 54, 4813 (1996).

[9] L. Blanchet, B. R. Iyer, C. M. Will, and A. G. Wiseman, Class. Quantum Grav. 13, 575 (1996).

[10] L. Blanchet, Phys. Rev. D 54, 1417 (1996).

[11] T. Tanaka, H. Tagoshi, and M. Sasaki, Prog. Theor. Phys. 96, 1087 (1996).

[12] C. Cutler et al., Phys. Rev. Lett. 70, 2984 (1993).

[13] E. Poisson, Phys. Rev. D 52, 5719 (1995).

[14] E. Poisson, Phys. Rev. D 55, 7980 (1997).

[15] The most accuracy-demanding case is the wave form emitted by a binary system made of a $\left(\sim 1.4 m_{\odot}\right)$ neutron star and a ( $\left.\gtrsim 10 m_{\odot}\right)$ black hole $[11,14]$.

[16] In the present work we shall only consider as parameters of the signal (besides an overall amplitude): two "kinematical" parameters $t_{c}, \Phi_{c}$ (a reference time and a reference phase), and two "dynamical" ones $m_{1}, m_{2}$ or equivalently $m, \eta$. Our intention here is to compare the performance of new approximants versus standard ones. Evidently, our results can then be trivially generalized by constructing the $P$-approximants of more general wave forms if the need arises.

[17] T. Damour and N. Deruelle, Phys. Lett. 87A, 81 (1981); C. R. Seances Acad. Sci., Ser. 2 293, 537 (1981); T. Damour, ibid. 294, 1355 (1982); in Gravitational Radiation, edited by N. Deruelle and T. Piran (North-Holland, Amsterdam, 1983), pp. 59-144

[18] We restrict our attention to circular(ized) systems.

[19] We henceforth use units such that $G=c=1$.

[20] For simplicity, we consider only diagonal approximants $(n, n)$.

[21] Optimization over phases $\phi$ is quite subtle and it is treated at length in Appendix B. 
[22] A loss in the overlap of $3.5 \%$ is equal to a reduction in the potential event rate by $10 \%$ (see Sec. V and Ref. [39] for details).

[23] The thresholds one may wish to retain to define the smallness of the biases will evidently depend on the context.

[24] But not sufficient, though one will, in practice, assume it to be so.

[25] L. Blanchet (unpublished); L. Blanchet, B. R. Iyer, and B. Joguet (in preparation); L. Blanchet, G. Faye, B. R. Iyer, B. Joguet, and B. Ponsot (work in progress); C. M. Will and M. E. Pati (work in progress); G. Schäfer and P. Jaranowski (work in progress).

[26] Note that using this rough approximation is consistent with our main purpose here which is to compare the usual postNewtonian approximants of the phase evolution to new approximants. We leave to future work the problem of deciding with what accuracy one should correspondingly model the tensorial amplitudes of each Fourier component of the signal.

[27] T. Damour and N. Deruelle, Ann. Inst. Henri Poincaré 44, 263 (1986); T. Damour and J. H. Taylor, Phys. Rev. D 45, 1840 (1992).

[28] The choice of $v_{1 \text { so }}^{A}$ does not matter as any change in this quantity can be reabsorbed in $t_{c}$ and $\Phi_{c}$. However, our approximants to $E_{A}^{\prime}(v)$ will have a zero (physically corresponding to the last stable orbit) and it will be natural and convenient to choose $v_{\text {lso }}^{A}$ as the location of this zero.

[29] C. Itzykson and J. B. Zuber, Quantum Field Theory (McGrawHill, New York, 1980), p. 83.

[30] L. Blanchet and T. Damour, Phys. Rev. D 37, 1410 (1988).

[31] In other words, we are assuming some minimum "structural stability", when turning on $\eta$.

[32] L. E. Kidder, C. M. Will, and A. G. Wiseman, Class. Quantum Grav. 9, L125 (1992); Phys. Rev. D 47, 3281 (1993).

[33] Note that we consistently label the successive approximants by the order in velocity. E.g. a $2 \mathrm{PN}$-accurate object has the label 4.

[34] N. Wex and G. Schäfer, Class. Quantum Grav. 10, 2729 (1993).

[35] It is to respect the consistency of the linearized gravitational equations $\left(\partial_{\nu} T^{\mu \nu}=0\right)$ that we had to take explicitly into ac- count the interaction accelerating the masses on their orbits.

[36] E.g., if one factorized out the log factors in the flux function of Eq. (4.1) as in Eq. (4.5),

$$
\begin{aligned}
F(v ; \eta=0)= & \frac{32}{5} \eta^{2} v^{10}\left[1+\ln \frac{v}{v_{\mathrm{lso}}(\eta)}\left(\sum_{k} \ell_{k} v^{k}\right)+\cdots\right] \\
& \times\left[\sum_{k} F_{k} v^{k}\right],
\end{aligned}
$$

then the corresponding coefficients $F_{k}$ are given by $F_{k}$ $=A_{k}, k=0 \cdots 5 ; F_{k}=A_{k}+B_{k} \ln v_{\text {lso }}, k=6 \cdots 11$. The $\ell_{k}$ are as given by Eq. (4.9).

[37] With the convention $\widetilde{g}(f) \equiv \int d t e^{2 \pi i f t} g(t)$.

[38] B. S. Sathyaprakash and S. V. Dhurandhar, Phys. Rev. D 44, 3819 (1991).

[39] B. J. Owen, Phys. Rev. D 53, 6749 (1996).

[40] B. J. Owen and B. S. Sathyaprakash (in preparation).

[41] C. Cutler and E. Flannagan, Phys. Rev. D 49, 2658 (1994).

[42] As $\mathcal{A}(h, g)$ is the infinite-dimensional generalization of the cosine of the angle between two unit vectors, we could define the angular distance between two normalized wave forms $(\|h\|=1=\|g\|)$ by $\theta=\arccos \mathcal{A}(h, g)$ and the corresponding chord by $\|h-g\|=2 \sin (\theta / 2)=\sqrt{2[1-\mathcal{A}(h, g)]}$.

[43] Since our $P_{4}^{3}$-approximant of flux has a pole in the region of interest, we have instead used the $P_{3}^{4}$-approximant in computing the overlaps and have not used the $P_{5}^{5}$-approximant (which also has a pole) at all.

[44] Actually several equivalent distance functions such as $\theta$ or $2 \sin (\theta / 2)$ as defined above [42].

[45] C. M. Bender and S. A. Orszag, Advanced Mathematical Methods for Scientists and Engineers (McGraw Hill, Singapore, 1984).

[46] The problem of phase maximization was first considered by Schutz for the case of two wave forms which both have the same shape: B. F. Schutz, in The Detection of Gravitational Radiation, edited by D. Blair (Cambridge, University Press, Cambridge, England, 1989), pp. 406-427 (also see Ref. [38]). Here we are addressing the problem of maximization over phase when the signal and the template wave forms belong to different post-Newtonian families. 